\title{
Hydrogen Bond Patterns of Dipyridone and Bis(Hydroxypyridinium) Cation
}

Midhun Mohan, ${ }^{a}$ Mohamed Essalhi, ${ }^{a}$ Sarah Zaye, ${ }^{a}$ Love Karan Rana, ${ }^{a}$ Thierry Maris ${ }^{\mathrm{b}}$ and Adam Duong*,a

${ }^{a}$ Département de Chimie, Biochimie et physique and Institut de Recherche sur l'Hydrogène, Université du Québec à Trois-Rivières, Trois-Rivières, Québec, G9A 5H7, Canada.

${ }^{b}$ Département de chimie, Université de Montréal, Montréal, Québec, H3T 1J4, Canada

*To whom correspondence should be addressed. E-mail : $\underline{\text { adam.duong@uqtr.ca }}$ 


\section{Table of Contents}

Figure S1. ${ }^{1} \mathrm{H}-\mathrm{NMR}$ spectrum of 2' recorded in DMSO- $d_{6}$ solution....................................S3

Figure S2. ${ }^{13} \mathrm{C}-\mathrm{NMR}$ spectrum of $\mathbf{2}^{\prime}$, recorded in DMSO- $d_{6}$ solution..................................S3

Figure S3. ${ }^{1} \mathrm{H}-\mathrm{NMR}$ spectrum of $\mathbf{2}$ recorded in DMSO- $d_{6}$ solution....................................S4

Figure S4. ${ }^{13} \mathrm{C}$-NMR spectrum of 2 recorded in DMSO- $d_{6}$ solution...................................S4

Figure S5. ${ }^{1} \mathrm{H}-\mathrm{NMR}$ spectrum of $\mathbf{3}$ ' recorded in DMSO- $d_{6}$ solution.....................................S5

Figure S6. ${ }^{13} \mathrm{C}-\mathrm{NMR}$ spectrum of $\mathbf{3}$ ' recorded in DMSO- $d_{6}$ solution...................................S5

Figure S7. ${ }^{1} \mathrm{H}-\mathrm{NMR}$ spectrum of $\mathbf{3}$ recorded in DMSO-d $\mathrm{d}_{6}$ solution....................................S6

Figure S8. ${ }^{13} \mathrm{C}-\mathrm{NMR}$ spectrum of $\mathbf{3}$ recorded in DMSO- $d_{6}$ solution....................................S6

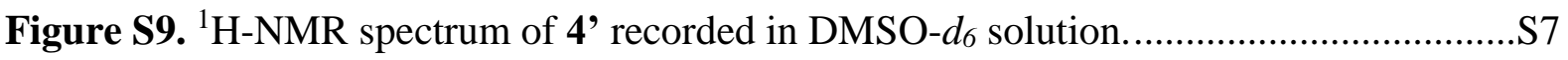

Figure S10. ${ }^{13} \mathrm{C}-\mathrm{NMR}$ spectrum of 4 ' recorded in DMSO- $d_{6}$ solution................................... 7

Figure S11. ${ }^{1} \mathrm{H}-\mathrm{NMR}$ spectrum of $\mathbf{4}$ recorded in TFA- $d$ solution. .....................................S8

Figure S12. ${ }^{13} \mathrm{C}-\mathrm{NMR}$ spectrum of 4 recorded in TFA- $d$ solution. ....................................S8

Figure S13. Thermal atomic displacement ellipsoid plot of 2 ..........................................S9

Figure S14. Thermal atomic displacement ellipsoid plot of 3......................................S9

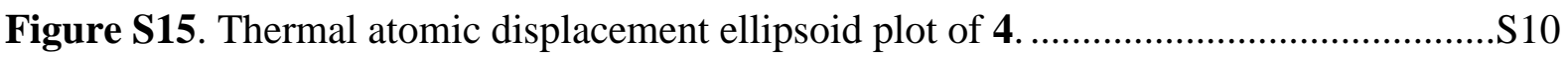

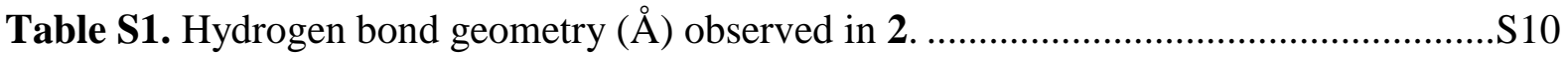

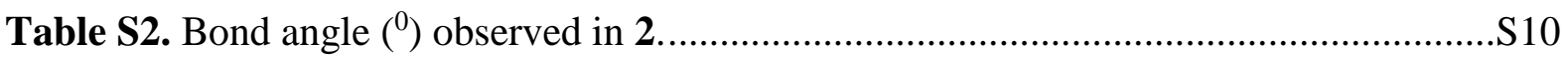

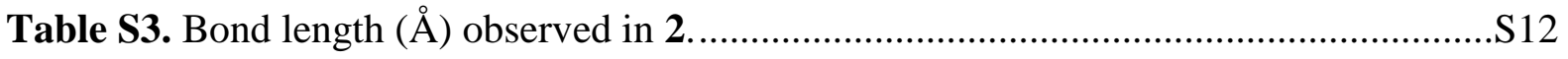

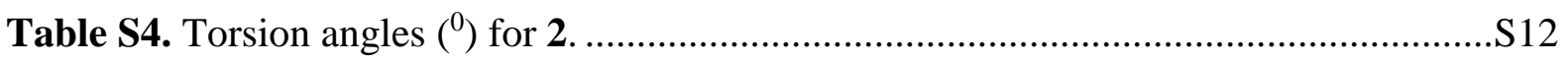

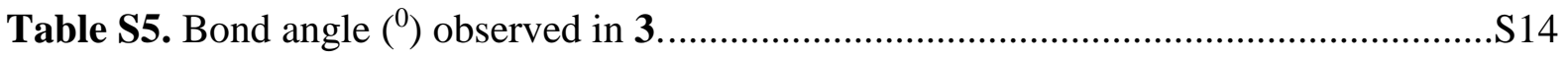

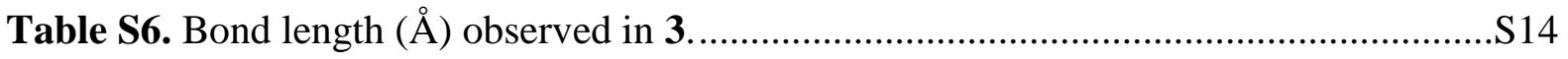

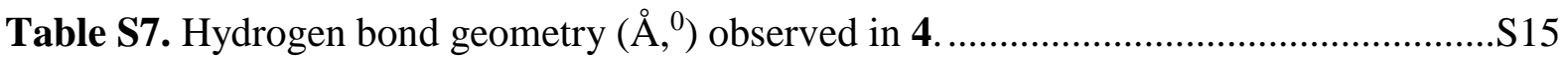

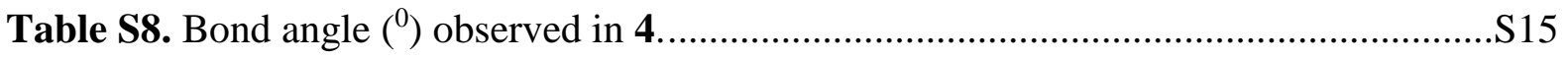

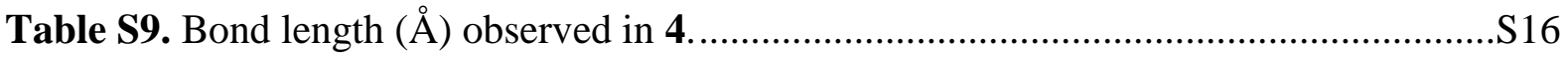

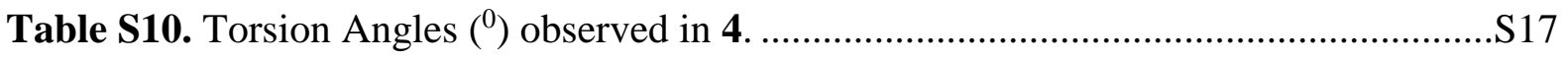

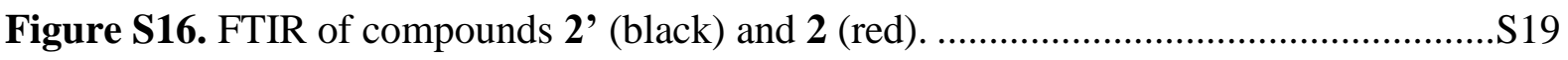

Figure S17. FTIR of compounds 3' (black) and $\mathbf{3}$ (red). .................................................. 19

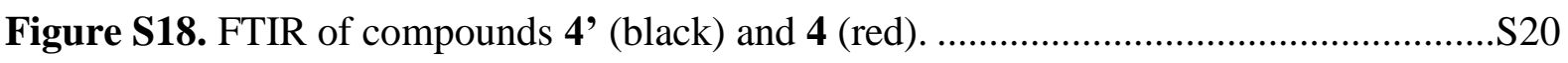

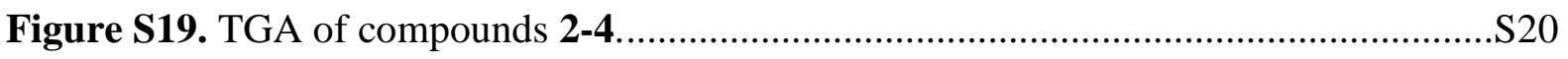




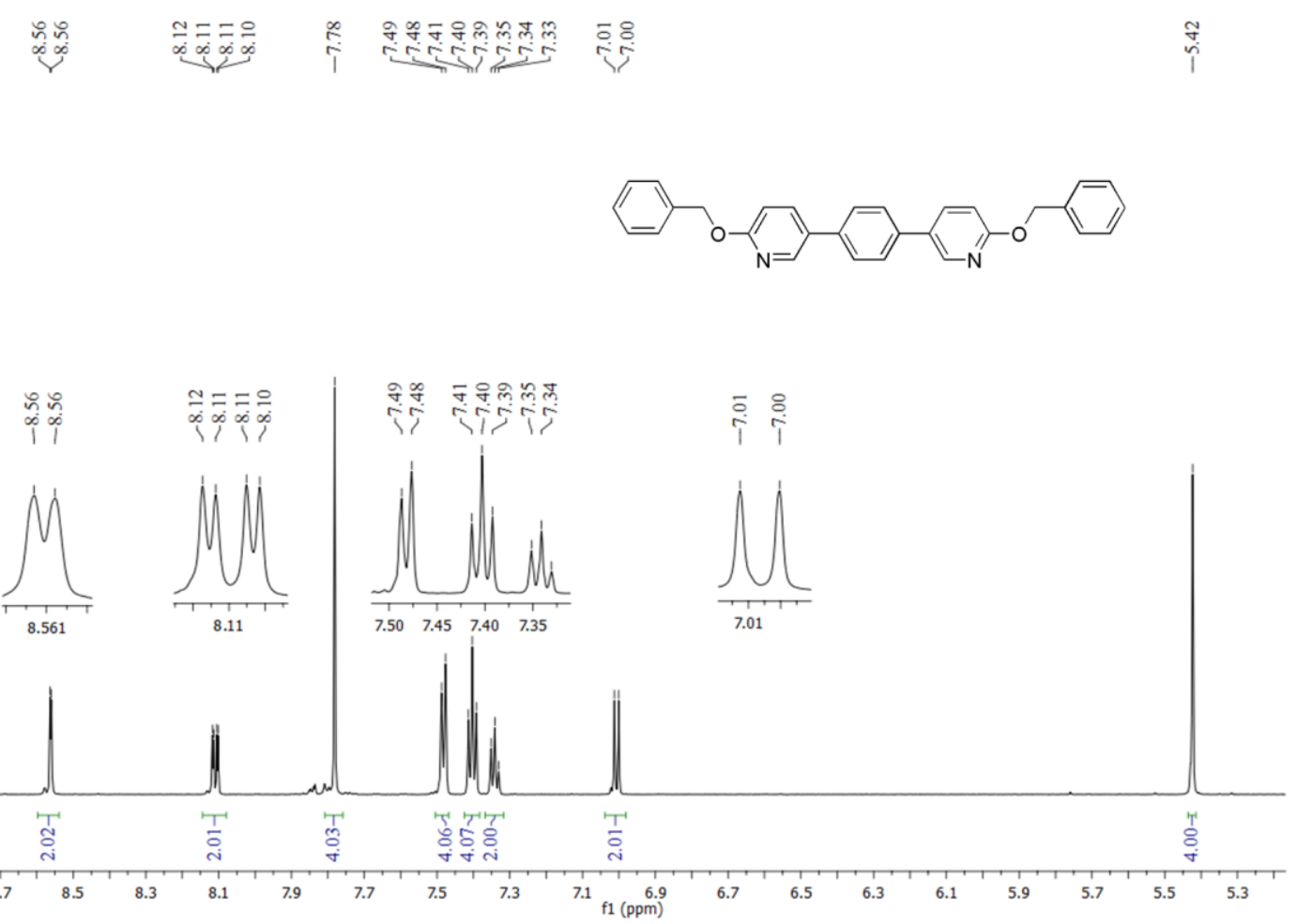

Figure S1. ${ }^{1} \mathrm{H}-\mathrm{NMR}$ spectrum of 2' recorded in DMSO- $d_{6}$ solution.

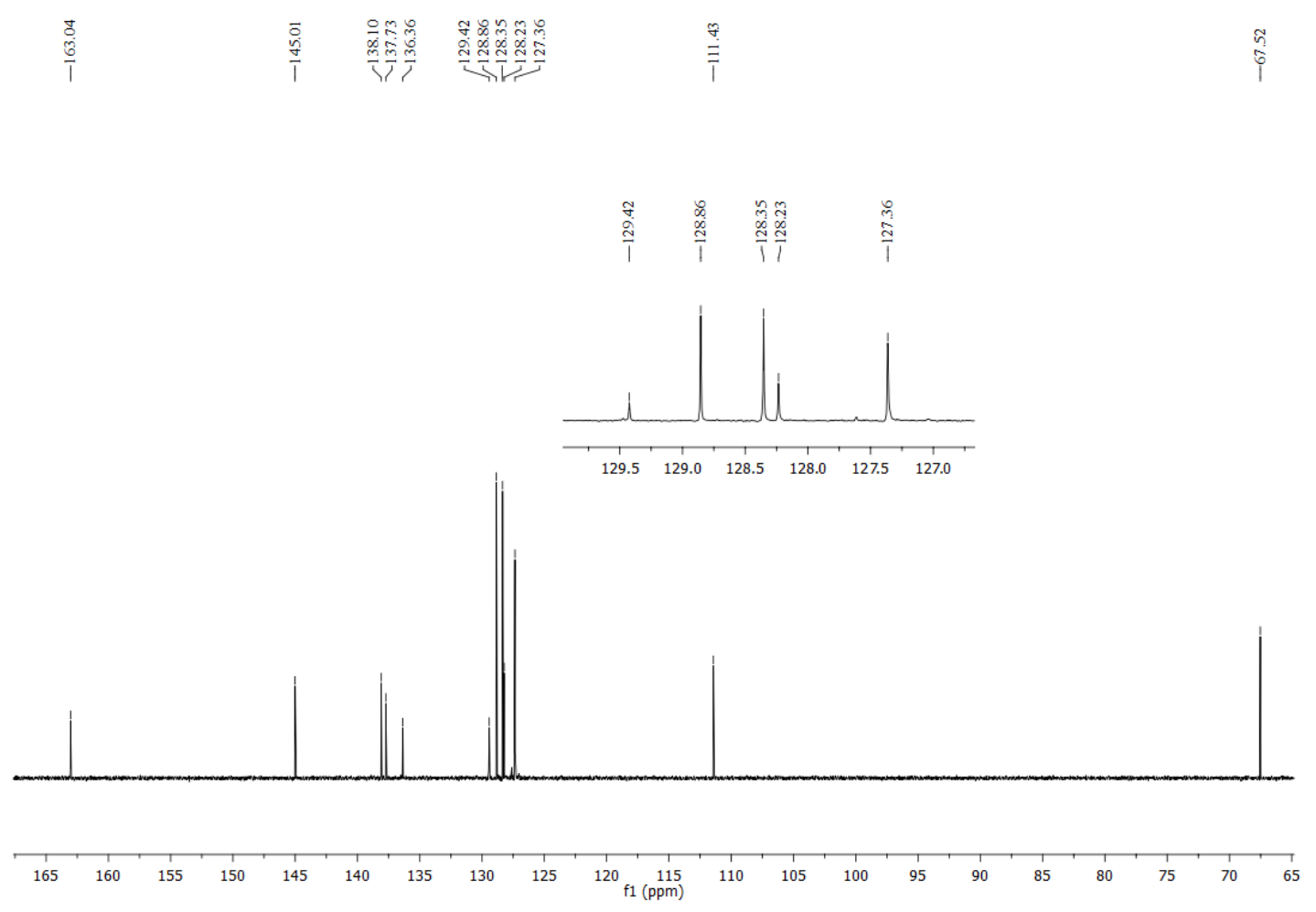

Figure S2. ${ }^{13} \mathrm{C}-\mathrm{NMR}$ spectrum of 2', recorded in DMSO- $d_{6}$ solution. 
<smiles>O=c1ccc(-c2ccc(-c3ccc(=O)[nH]c3)cc2)c[nH]1</smiles>

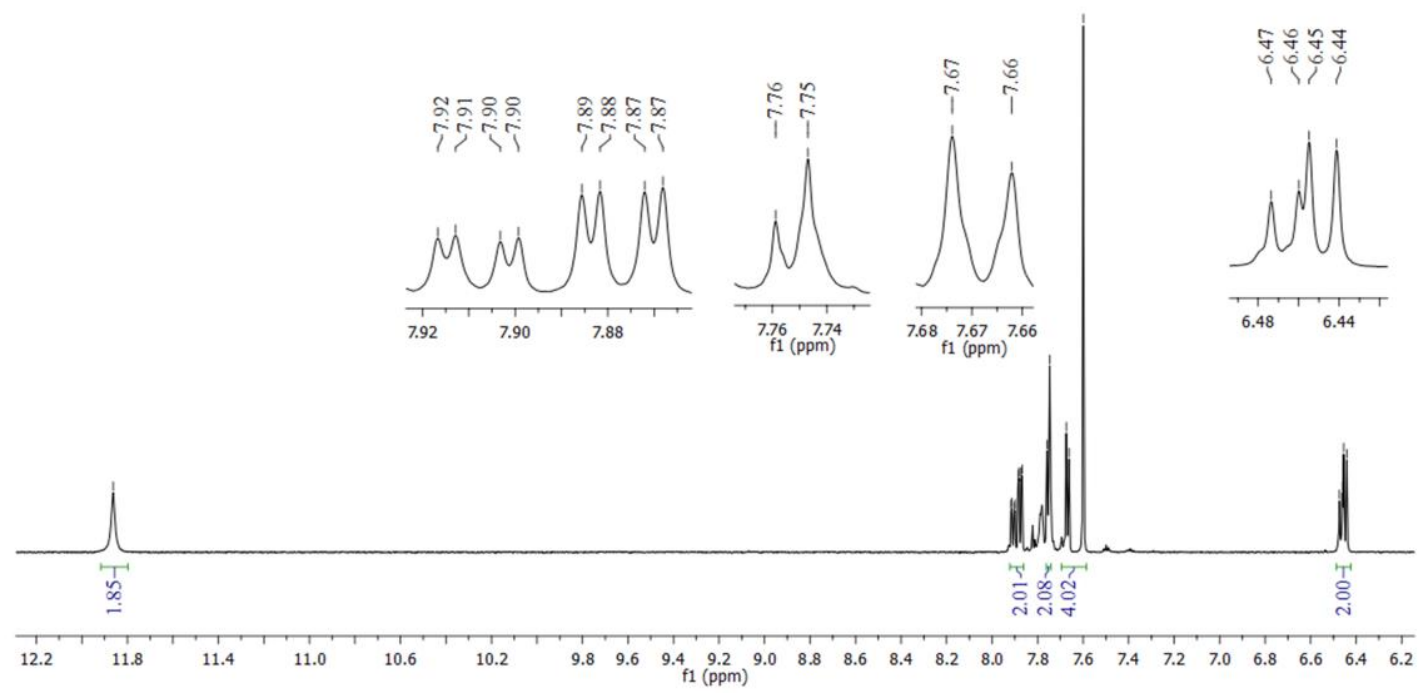

Figure S3. ${ }^{1} \mathrm{H}-\mathrm{NMR}$ spectrum of 2 recorded in DMSO- $d_{6}$ solution.

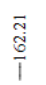
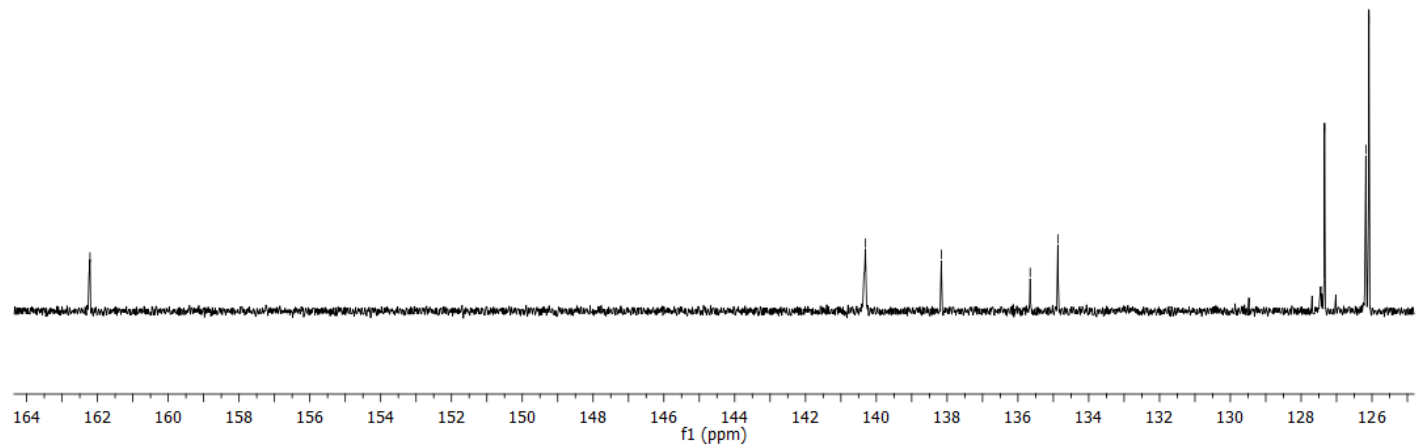

Figure S4. ${ }^{13} \mathrm{C}-\mathrm{NMR}$ spectrum of 2 recorded in DMSO- $d_{6}$ solution. 


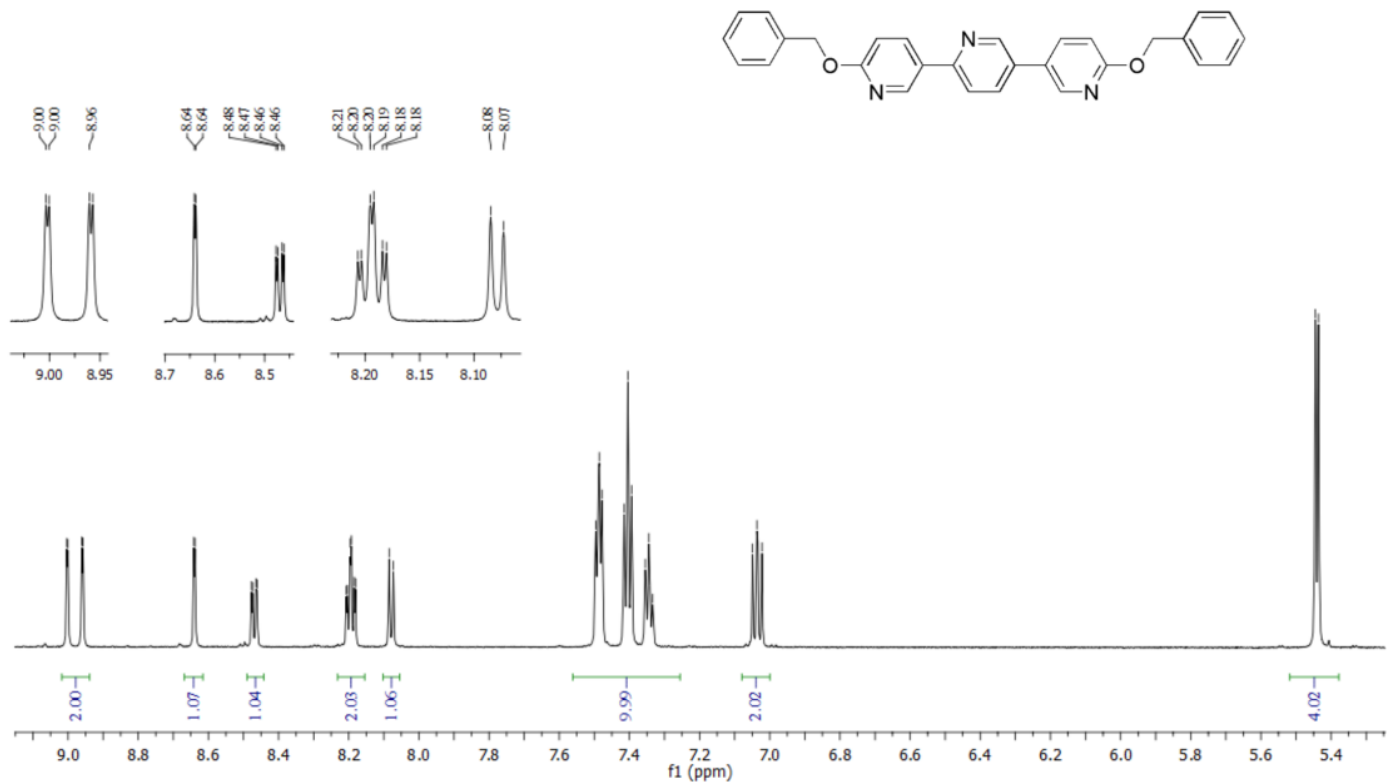

Figure S5. ${ }^{1} \mathrm{H}-\mathrm{NMR}$ spectrum of $3{ }^{\prime}$ recorded in DMSO- $d_{6}$ solution.

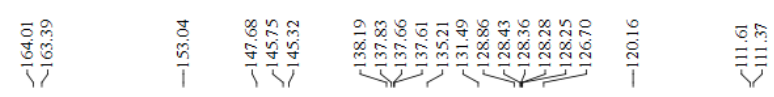

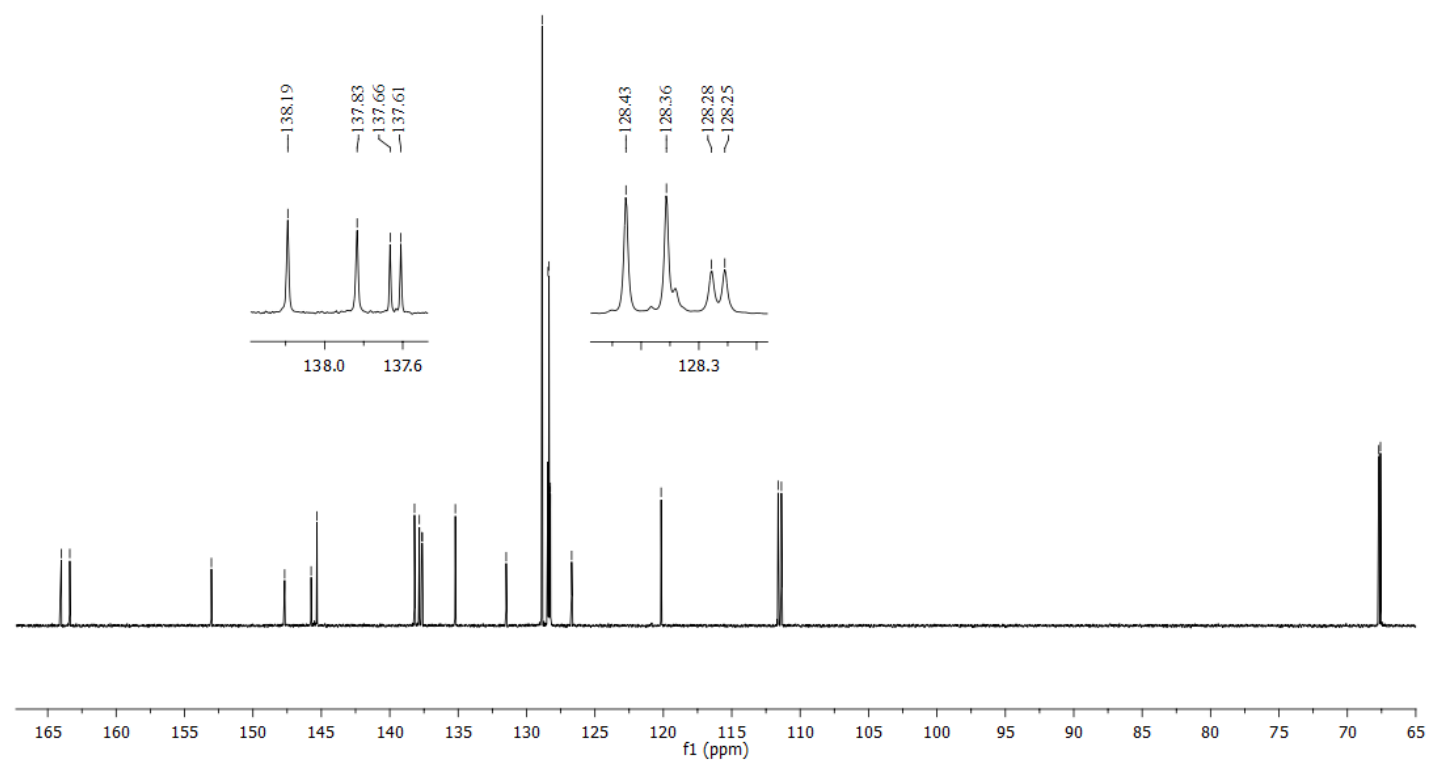

Figure S6. ${ }^{13} \mathrm{C}-\mathrm{NMR}$ spectrum of $\mathbf{3}$ ' recorded in DMSO- $d_{6}$ solution. 
<smiles>O=c1ccc(-c2ccc(-c3ccc(=O)[nH]c3)nc2)c[nH]1</smiles>

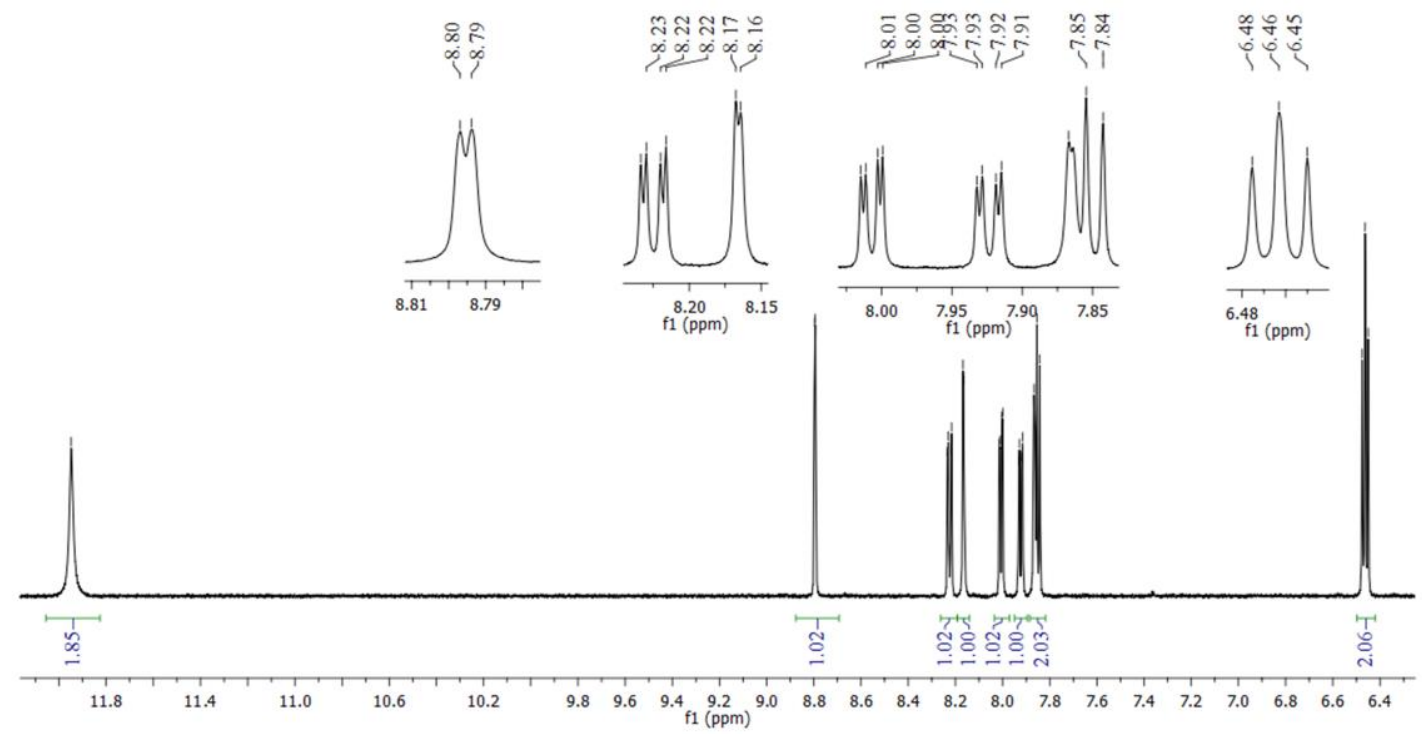

Figure S7. ${ }^{1} \mathrm{H}-\mathrm{NMR}$ spectrum of 3 recorded in DMSO-d 6 solution.

\begin{tabular}{|c|c|c|c|c|}
\hline 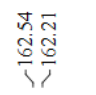 & $\frac{\tilde{6}}{\frac{\pi}{n}}$ & $\begin{array}{l}\text { ले } \\
\text { ơ } \\
\frac{0}{1}\end{array}$ & 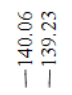 & 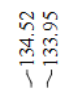 \\
\hline
\end{tabular}

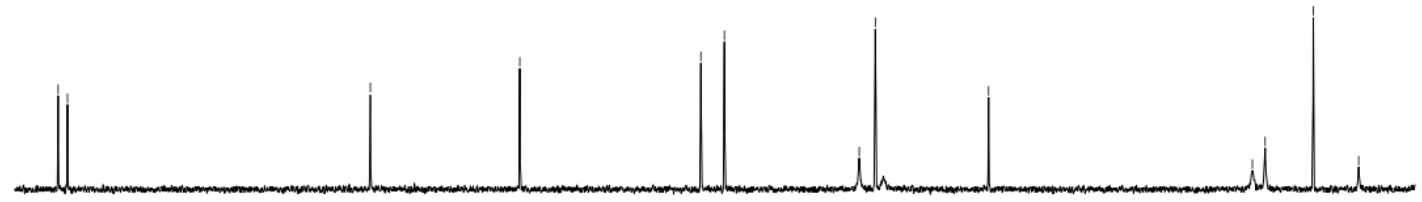

$\begin{array}{lllllllllllllllllllllllllllllllll}164 & 162 & 160 & 158 & 156 & 154 & 152 & 150 & 148 & 146 & 144 & 142 & 140 & 138 & 136 & 134 & 132 & 130 & 128 & 126 & 124 & 122 & 120 & 118 & 116\end{array}$

Figure S8. ${ }^{13} \mathrm{C}-\mathrm{NMR}$ spectrum of 3 recorded in DMSO- $d_{6}$ solution. 


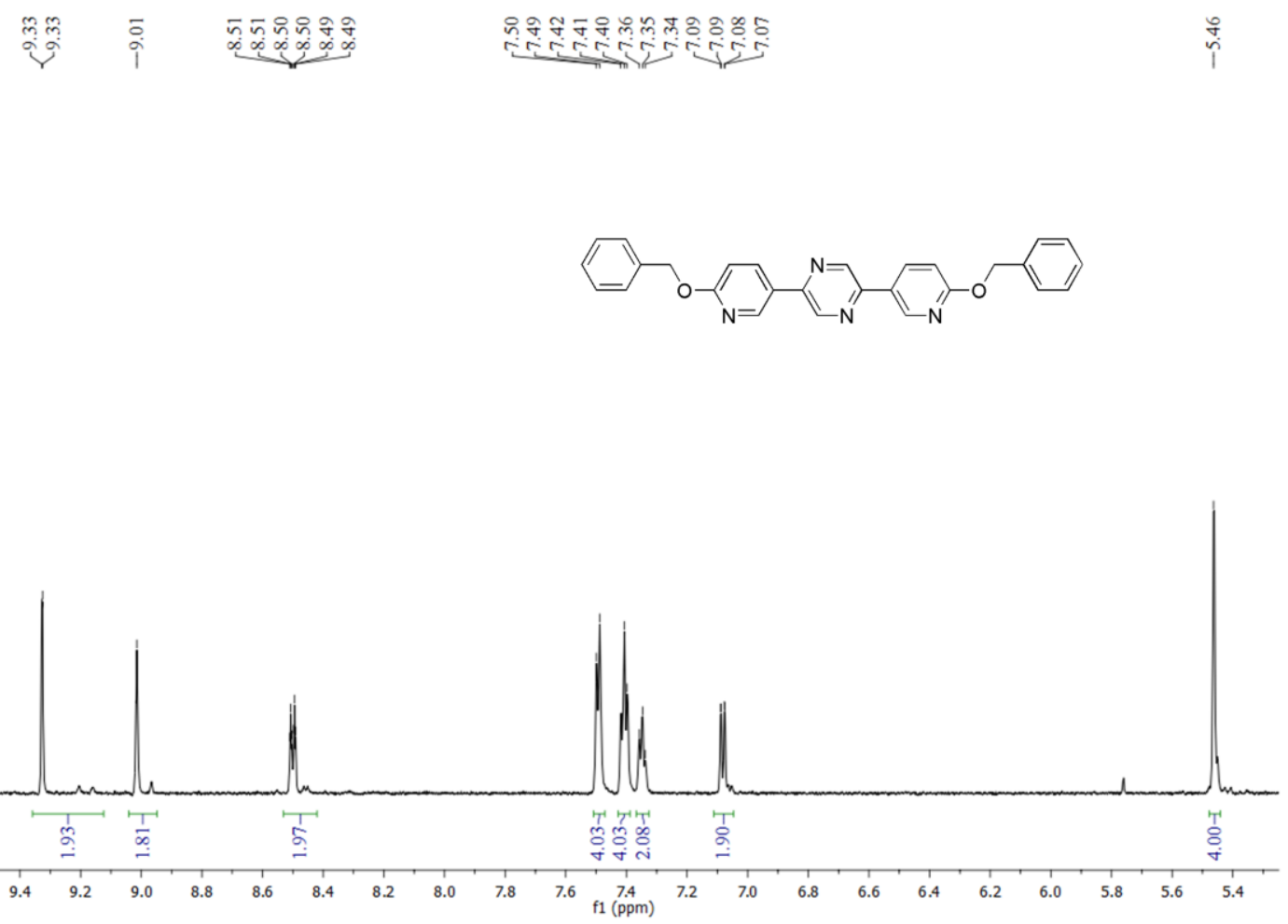

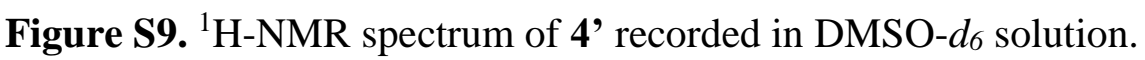
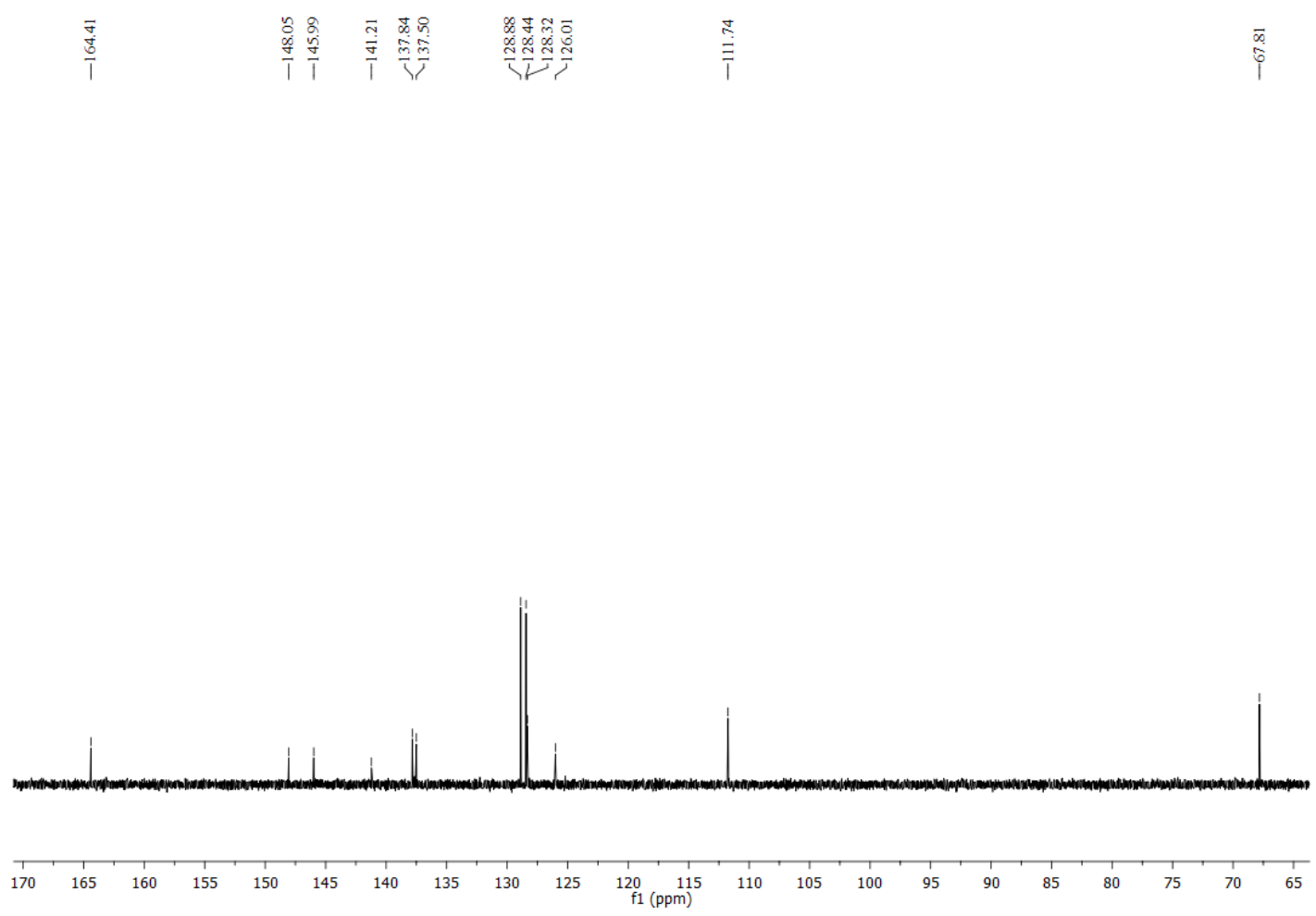

Figure S10. ${ }^{13} \mathrm{C}-\mathrm{NMR}$ spectrum of 4' recorded in DMSO- $d_{6}$ solution. 


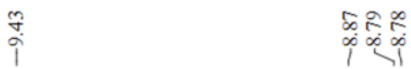
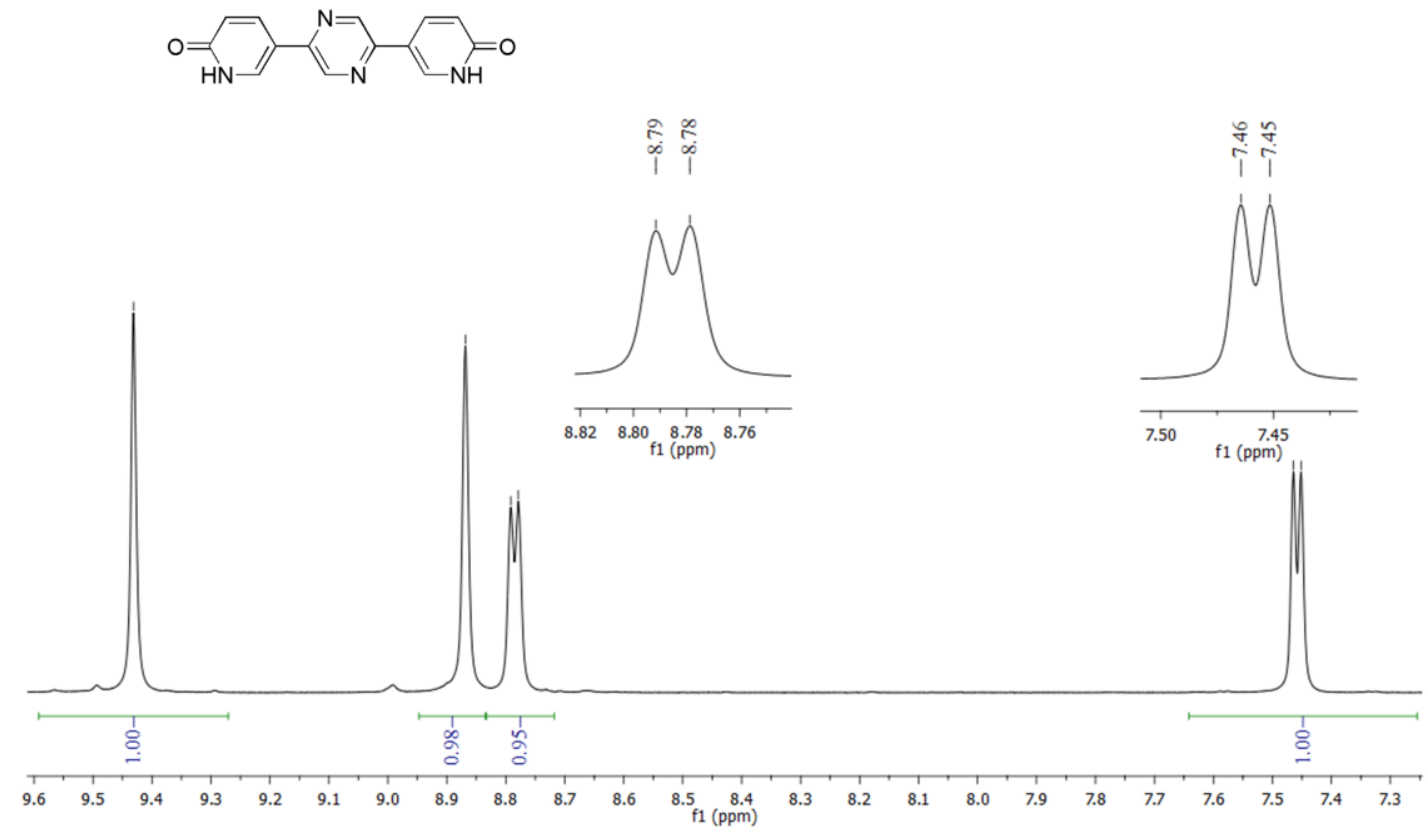

Figure S11. ${ }^{1} \mathrm{H}-\mathrm{NMR}$ spectrum of $\mathbf{4}$ recorded in TFA- $d$ solution.

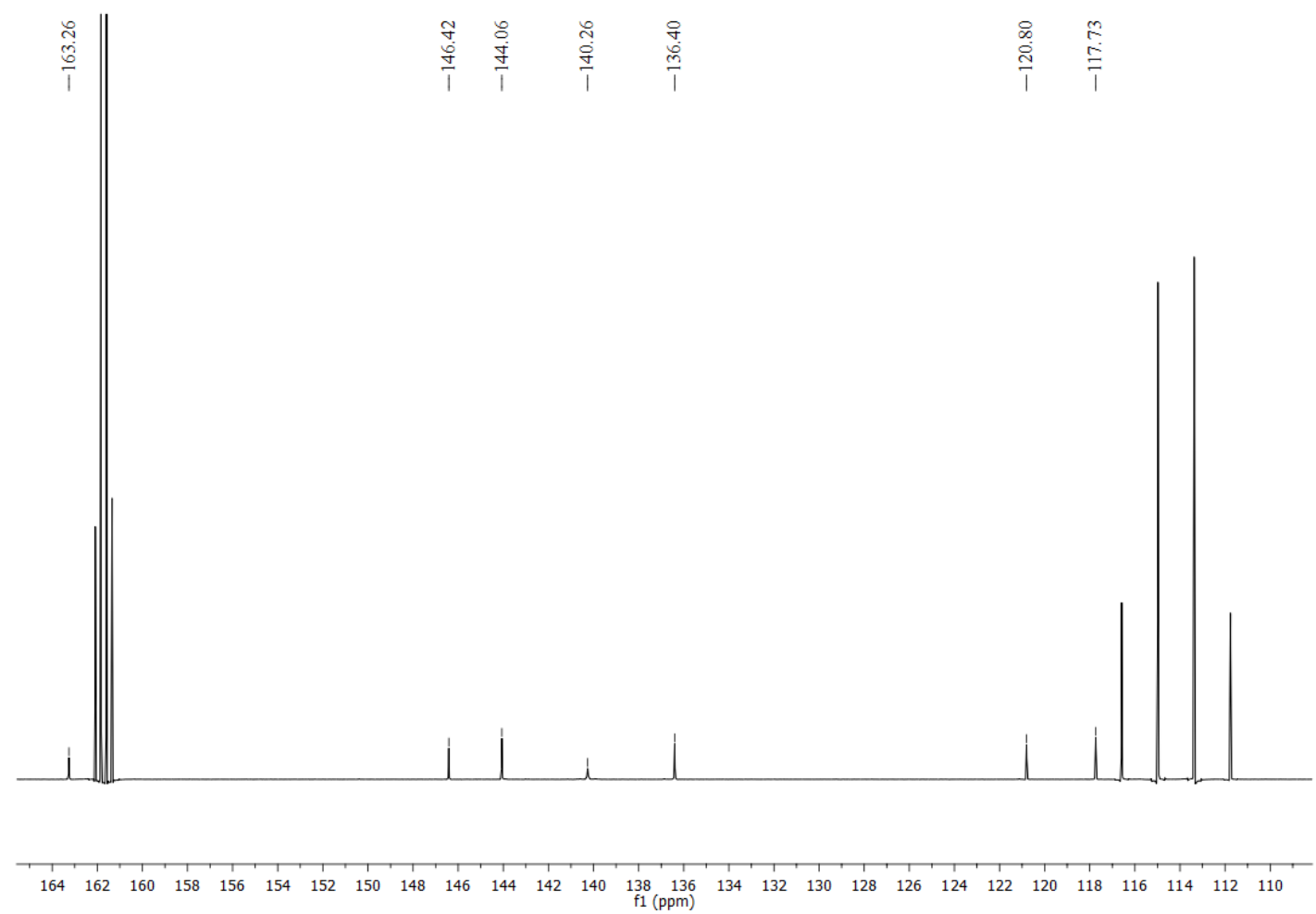

Figure S12. ${ }^{13} \mathrm{C}-\mathrm{NMR}$ spectrum of $\mathbf{4}$ recorded in TFA- $d$ solution. 


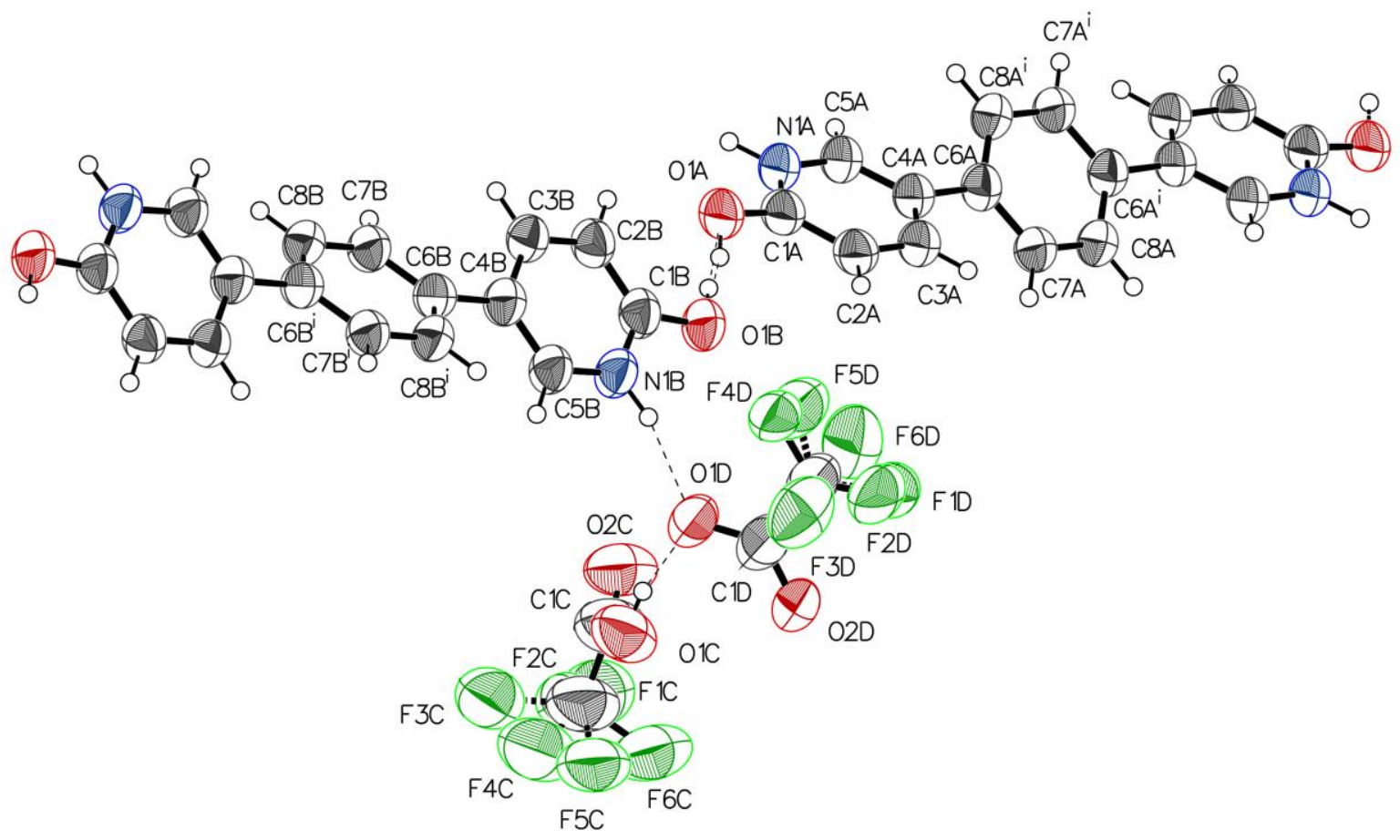

Figure S13. Thermal atomic displacement ellipsoid plot of $\mathbf{2}$. Thermal atomic displacement ellipsoid plot of 2 . The ellipsoids of non-hydrogen atoms are drawn at 50\% probability level, hydrogen atoms are represented by a sphere of arbitrary size, and hydrogen bonds are represented by dotted lines.

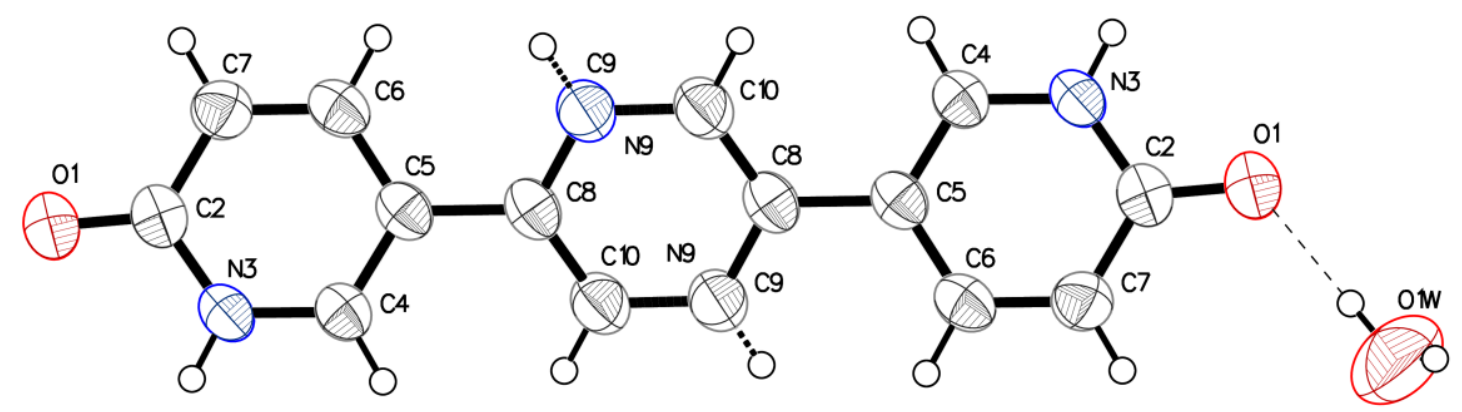

Figure S14. Thermal atomic displacement ellipsoid plot of 3. The ellipsoids of non-hydrogen atoms are drawn at $50 \%$ probability level, hydrogen atoms are represented by a sphere of arbitrary size, and hydrogen bonds are represented by dotted lines. 


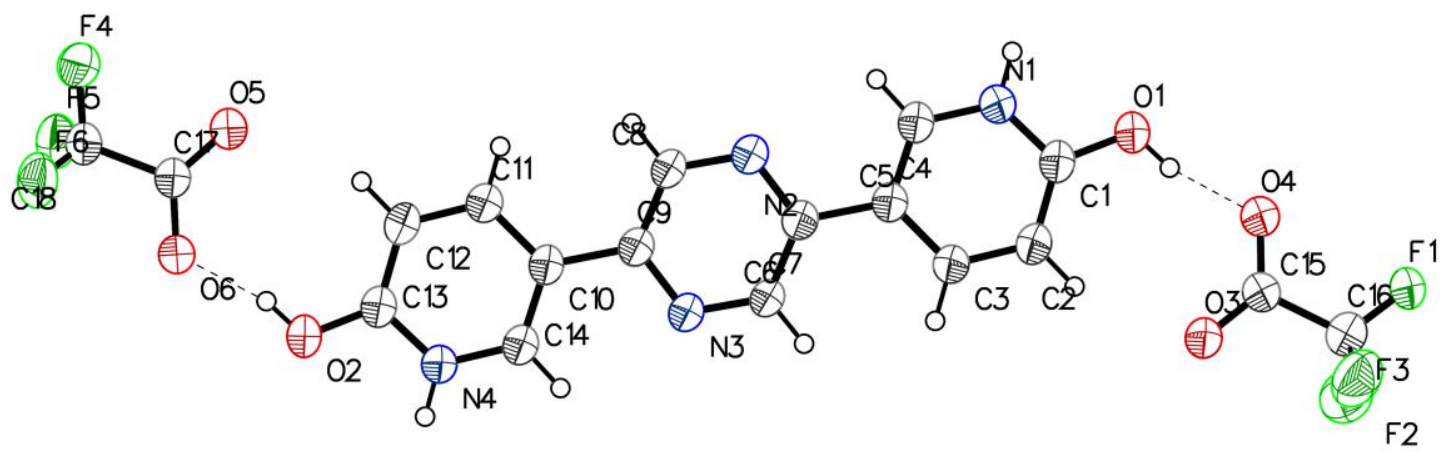

Figure S15. Thermal atomic displacement ellipsoid plot of 4 . The ellipsoids of non-hydrogen atoms are drawn at $50 \%$ probability level, hydrogen atoms are represented by a sphere of arbitrary size, and hydrogen bonds are represented by dotted lines.

Table S1. Hydrogen bond geometry ( $\mathrm{A})$ observed in 2 .

\begin{tabular}{lllll}
$\boldsymbol{D}-\boldsymbol{H} \cdots \boldsymbol{A}$ & $\boldsymbol{D}-\boldsymbol{H}$ & $\boldsymbol{H} \cdots \boldsymbol{A}$ & $\boldsymbol{D} \cdots \boldsymbol{A}$ & $\boldsymbol{D}-\boldsymbol{H} \cdots \boldsymbol{A}$ \\
\hline O1C-H1C $\cdots$ O1D & 0.84 & 1.65 & $2.474(4)$ & 167.1 \\
O1B-H1B $\cdots$ O1A & 0.84 & 1.59 & $2.431(3)$ & 175.1 \\
N1B-H1BA $\cdots$ F4D & $0.91(3)$ & $2.44(3)$ & $3.011(12)$ & $120.8(19)$ \\
N1B-H1BA $\cdots$ O1D & $0.91(3)$ & $1.90(3)$ & $2.789(3)$ & $167(2)$ \\
O1A-H1A $\cdots$ O1B & 0.84 & 1.61 & $2.431(3)$ & 164.0 \\
N1A-H1AA $\cdots$ O2D & $0.95(3)$ & $1.84(3)$ & $2.784(3)$ & $173(2)$ \\
\hline
\end{tabular}

Symmetry codes: (i) $+\mathrm{x}, 1+\mathrm{y},+\mathrm{z}$.

Table S2. Bond angle $\left({ }^{0}\right)$ observed in 2.

\begin{tabular}{llll} 
Atom & Atom & Atom & Angle/ \\
\hline O1C & C1C & C2C & $113.7(5)$ \\
O2C & C1C & O1C & $129.2(5)$ \\
O2C & C1C & C2C & $117.0(6)$ \\
F2C & F3C & $93.6(6)$ \\
F2C & F5C & $129.5(8)$ \\
F2C & C2C & C1C & $117.0(6)$ \\
F4C & C2C & F1C & $127.2(8)$ \\
F4C & C2C & F6C & $101.6(7)$ \\
F4C & C2C & F3C & $121.2(9)$ \\
F5C & C2C & C1C & $90.0(8)$ \\
F5C & C2C & F1C & $112.7(6)$ \\
F6C & C2C & C1C & $80.1(8)$ \\
F6C & C2C & F1C & $106.9(6)$ \\
C1C & C2C & F3C & $107.9(7)$ \\
C1C & C2C & C2D & $95.6(6)$ \\
O1D & C2C & O1D & $114.7(3)$ \\
O2D & C1D & C2D & $129.2(4)$ \\
O2D & C1D & C1D &
\end{tabular}




\begin{tabular}{|c|c|c|c|}
\hline F1D & C2D & F3D & $98.9(12)$ \\
\hline F1D & $\mathrm{C} 2 \mathrm{D}$ & C1D & $115.9(11)$ \\
\hline $\mathrm{F} 2 \mathrm{D}$ & $\mathrm{C} 2 \mathrm{D}$ & F4D & $121.8(13)$ \\
\hline $\mathrm{F} 2 \mathrm{D}$ & $\mathrm{C} 2 \mathrm{D}$ & C1D & $123.1(12)$ \\
\hline $\mathrm{F} 2 \mathrm{D}$ & $\mathrm{C} 2 \mathrm{D}$ & F6D & $88.4(13)$ \\
\hline $\mathrm{F} 4 \mathrm{D}$ & $\mathrm{C} 2 \mathrm{D}$ & C1D & $114.9(6)$ \\
\hline $\mathrm{F} 4 \mathrm{D}$ & $\mathrm{C} 2 \mathrm{D}$ & F6D & $93.7(6)$ \\
\hline F5D & $\mathrm{C} 2 \mathrm{D}$ & F1D & $108.7(13)$ \\
\hline F5D & $\mathrm{C} 2 \mathrm{D}$ & F3D & $104.4(9)$ \\
\hline F5D & $\mathrm{C} 2 \mathrm{D}$ & C1D & $122.7(8)$ \\
\hline C1D & $\mathrm{C} 2 \mathrm{D}$ & F3D & $102.3(4)$ \\
\hline C1D & $\mathrm{C} 2 \mathrm{D}$ & F6D & $92.7(4)$ \\
\hline $\mathrm{C} 1 \mathrm{~B}$ & $\mathrm{~N} 1 \mathrm{~B}$ & C5B & $123.6(2)$ \\
\hline O1B & $\mathrm{C} 1 \mathrm{~B}$ & N1B & $116.8(2)$ \\
\hline O1B & $\mathrm{C} 1 \mathrm{~B}$ & $\mathrm{C} 2 \mathrm{~B}$ & $126.0(2)$ \\
\hline N1B & $\mathrm{C} 1 \mathrm{~B}$ & $\mathrm{C} 2 \mathrm{~B}$ & $117.1(2)$ \\
\hline $\mathrm{C} 3 \mathrm{~B}$ & $\mathrm{C} 2 \mathrm{~B}$ & $\mathrm{C} 1 \mathrm{~B}$ & $119.8(2)$ \\
\hline $\mathrm{C} 2 \mathrm{~B}$ & C3B & C4B & $121.8(2)$ \\
\hline $\mathrm{C} 3 \mathrm{~B}$ & $\mathrm{C} 4 \mathrm{~B}$ & C6B & $122.0(2)$ \\
\hline C5B & C4B & C3B & $117.0(2)$ \\
\hline C5B & $\mathrm{C} 4 \mathrm{~B}$ & C6B & $121.0(2)$ \\
\hline N1B & $\mathrm{C} 5 \mathrm{~B}$ & $\mathrm{C} 4 \mathrm{~B}$ & $120.6(2)$ \\
\hline C7B & C6B & C4B & $121.2(2)$ \\
\hline C7B & C6B & $\mathrm{C} 8 \mathrm{~B}^{\mathrm{i}}$ & $117.9(2)$ \\
\hline $\mathrm{C} 8 \mathrm{~B}^{\mathrm{i}}$ & C6B & $\mathrm{C} 4 \mathrm{~B}$ & $120.9(2)$ \\
\hline $\mathrm{C} 8 \mathrm{~B}$ & C7B & C6B & $121.2(2)$ \\
\hline C7B & $\mathrm{C} 8 \mathrm{~B}$ & C6 $B^{i}$ & $120.9(2)$ \\
\hline C5A & $\mathrm{N} 1 \mathrm{~A}$ & $\mathrm{C} 1 \mathrm{~A}$ & $123.1(2)$ \\
\hline O1A & $\mathrm{C} 1 \mathrm{~A}$ & $\mathrm{~N} 1 \mathrm{~A}$ & $117.1(2)$ \\
\hline O1A & $\mathrm{C} 1 \mathrm{~A}$ & $\mathrm{C} 2 \mathrm{~A}$ & $126.4(2)$ \\
\hline N1A & $\mathrm{C} 1 \mathrm{~A}$ & $\mathrm{C} 2 \mathrm{~A}$ & $116.5(2)$ \\
\hline $\mathrm{C} 3 \mathrm{~A}$ & $\mathrm{C} 2 \mathrm{~A}$ & $\mathrm{C} 1 \mathrm{~A}$ & $120.7(2)$ \\
\hline $\mathrm{C} 2 \mathrm{~A}$ & $\mathrm{C} 3 \mathrm{~A}$ & $\mathrm{C} 4 \mathrm{~A}$ & $121.4(2)$ \\
\hline $\mathrm{C} 3 \mathrm{~A}$ & $\mathrm{C} 4 \mathrm{~A}$ & C6A & $122.1(2)$ \\
\hline $\mathrm{C} 5 \mathrm{~A}$ & $\mathrm{C} 4 \mathrm{~A}$ & $\mathrm{C} 3 \mathrm{~A}$ & $116.6(2)$ \\
\hline $\mathrm{C} 5 \mathrm{~A}$ & $\mathrm{C} 4 \mathrm{~A}$ & C6A & $121.3(2)$ \\
\hline N1A & C5A & $\mathrm{C} 4 \mathrm{~A}$ & $121.8(2)$ \\
\hline C7A & C6A & $\mathrm{C} 4 \mathrm{~A}$ & $120.6(2)$ \\
\hline C7A & C6A & $\mathrm{C} 8 \mathrm{~A}^{\mathrm{ii}}$ & $118.4(2)$ \\
\hline $\mathrm{C} 8 \mathrm{~A}^{\mathrm{ii}}$ & C6A & $\mathrm{C} 4 \mathrm{~A}$ & $121.0(2)$ \\
\hline $\mathrm{C} 8 \mathrm{~A}$ & C7A & C6A & $121.3(2)$ \\
\hline C7A & C8A & C6 $\mathrm{A}^{\mathrm{ii}}$ & $120.3(2)$ \\
\hline
\end{tabular}

Symmetry codes: (i) $-\mathrm{x}+3,-\mathrm{y}+2,-\mathrm{z}+1$; (ii) $-\mathrm{x},-\mathrm{y}+2,-\mathrm{z}$. 
Table S3. Bond length $(\AA)$ observed in 2.

\begin{tabular}{|c|c|c|c|}
\hline Atom-Atom & Length $[\AA]$ & Atom-Atom & $\begin{array}{l}\text { Length } \\
{[\AA]}\end{array}$ \\
\hline $\mathrm{F} 1 \mathrm{C}-\mathrm{C} 2 \mathrm{C}$ & $1.622(16)$ & $\mathrm{C} 3 \mathrm{~B}-\mathrm{C} 4 \mathrm{~B}$ & $1.414(3)$ \\
\hline $\mathrm{F} 2 \mathrm{C}-\mathrm{C} 2 \mathrm{C}$ & $1.216(11)$ & $\mathrm{C} 4 \mathrm{~B}-\mathrm{C} 5 \mathrm{~B}$ & $1.368(3)$ \\
\hline $\mathrm{F} 3 \mathrm{C}-\mathrm{C} 2 \mathrm{C}$ & $1.647(11)$ & $\mathrm{C} 4 \mathrm{~B}-\mathrm{C} 6 \mathrm{~B}$ & $1.485(3)$ \\
\hline $\mathrm{F} 4 \mathrm{C}-\mathrm{C} 2 \mathrm{C}$ & $1.216(10)$ & C5B-H5B & 0.9500 \\
\hline $\mathrm{F} 5 \mathrm{C}-\mathrm{C} 2 \mathrm{C}$ & $1.265(10)$ & C6B-C7B & $1.395(4)$ \\
\hline $\mathrm{F} 6 \mathrm{C}-\mathrm{C} 2 \mathrm{C}$ & $1.428(12)$ & $\mathrm{C} 6 \mathrm{~B}-\mathrm{C} 8 \mathrm{~B}^{\mathrm{i}}$ & $1.399(4)$ \\
\hline $\mathrm{O} 1 \mathrm{C}-\mathrm{H} 1 \mathrm{C}$ & 0.8400 & C7B-H7B & 0.9500 \\
\hline $\mathrm{O} 1 \mathrm{C}-\mathrm{C} 1 \mathrm{C}$ & $1.309(7)$ & $\mathrm{C} 7 \mathrm{~B}-\mathrm{C} 8 \mathrm{~B}$ & $1.379(3)$ \\
\hline $\mathrm{O} 2 \mathrm{C}-\mathrm{C} 1 \mathrm{C}$ & $1.184(6)$ & C8B-H8B & 0.9500 \\
\hline $\mathrm{C} 1 \mathrm{C}-\mathrm{C} 2 \mathrm{C}$ & $1.548(8)$ & $\mathrm{O} 1 \mathrm{~A}-\mathrm{H} 1 \mathrm{~A}$ & 0.8400 \\
\hline F1D-C2D & $1.31(2)$ & $\mathrm{O} 1 \mathrm{~A}-\mathrm{C} 1 \mathrm{~A}$ & $1.264(3)$ \\
\hline F2D-C2D & $1.135(19)$ & N1A-H1AA & $0.95(3)$ \\
\hline F3D-C2D & $1.739(6)$ & $\mathrm{N} 1 \mathrm{~A}-\mathrm{C} 1 \mathrm{~A}$ & $1.365(4)$ \\
\hline F4D-C2D & $1.315(13)$ & $\mathrm{N} 1 \mathrm{~A}-\mathrm{C} 5 \mathrm{~A}$ & $1.354(3)$ \\
\hline F5D-C2D & $1.266(18)$ & $\mathrm{C} 1 \mathrm{~A}-\mathrm{C} 2 \mathrm{~A}$ & $1.412(4)$ \\
\hline O1D-C1D & $1.252(4)$ & $\mathrm{C} 2 \mathrm{~A}-\mathrm{H} 2 \mathrm{~A}$ & 0.9500 \\
\hline $\mathrm{O} 2 \mathrm{D}-\mathrm{C} 1 \mathrm{D}$ & $1.208(4)$ & $\mathrm{C} 2 \mathrm{~A}-\mathrm{C} 3 \mathrm{~A}$ & $1.367(3)$ \\
\hline C1D-C2D & $1.503(7)$ & $\mathrm{C} 3 \mathrm{~A}-\mathrm{H} 3 \mathrm{~A}$ & 0.9500 \\
\hline C2D-F6D & $1.740(7)$ & $\mathrm{C} 3 \mathrm{~A}-\mathrm{C} 4 \mathrm{~A}$ & $1.410(4)$ \\
\hline O1B-H1B & 0.8400 & $\mathrm{C} 4 \mathrm{~A}-\mathrm{C} 5 \mathrm{~A}$ & $1.372(3)$ \\
\hline $\mathrm{O} 1 \mathrm{~B}-\mathrm{C} 1 \mathrm{~B}$ & $1.289(3)$ & $\mathrm{C} 4 \mathrm{~A}-\mathrm{C} 6 \mathrm{~A}$ & $1.485(3)$ \\
\hline N1B-H1BA & $0.91(3)$ & C5A-H5A & 0.9500 \\
\hline $\mathrm{N} 1 \mathrm{~B}-\mathrm{C} 1 \mathrm{~B}$ & $1.352(3)$ & C6A-C7A & $1.398(3)$ \\
\hline $\mathrm{N} 1 \mathrm{~B}-\mathrm{C} 5 \mathrm{~B}$ & $1.362(3)$ & $\mathrm{C} 6 \mathrm{~A}-\mathrm{C} 8 \mathrm{~A}^{\mathrm{ii}}$ & $1.409(3)$ \\
\hline $\mathrm{C} 1 \mathrm{~B}-\mathrm{C} 2 \mathrm{~B}$ & $1.412(4)$ & C7A-H7A & 0.9500 \\
\hline $\mathrm{C} 2 \mathrm{~B}-\mathrm{H} 2 \mathrm{~B}$ & 0.9500 & $\mathrm{C} 7 \mathrm{~A}-\mathrm{C} 8 \mathrm{~A}$ & $1.387(3)$ \\
\hline $\mathrm{C} 2 \mathrm{~B}-\mathrm{C} 3 \mathrm{~B}$ & $1.361(3)$ & C8A-H8A & 0.9500 \\
\hline $\mathrm{C} 3 \mathrm{~B}-\mathrm{H} 3 \mathrm{~B}$ & 0.9500 & & \\
\hline
\end{tabular}

Symmetry codes: (i) $-\mathrm{x}+3,-\mathrm{y}+2,-\mathrm{z}+1$; (ii) $-\mathrm{x},-\mathrm{y}+2,-\mathrm{z}$.

Table S4. Torsion angles $\left({ }^{0}\right)$ for 2.

\begin{tabular}{lllll} 
A & B & C & D & Angle ${ }^{\circ}$ \\
\hline O1C & C1C & C2C & F1C & $-162.6(7)$ \\
O1C & C1C & C2C & F2C & $174.8(6)$ \\
O1C & C1C & C2C & F3C & $77.8(6)$ \\
O1C & C1C & C2C & F4C & $37.6(11)$ \\
O1C & C1C & C2C & F5C & $-14.6(12)$ \\
O1C & C1C & C2C & F6C & $-77.8(9)$ \\
O2C & C1C & C2C & F1C & $14.9(10)$ \\
O2C & C1C & C2C & F2C & $-7.7(11)$ \\
O2C & C1C & C2C & F3C & $-104.6(7)$ \\
O2C & C1C & C2C & F4C & $-144.8(9)$
\end{tabular}




\begin{tabular}{|c|c|c|c|c|}
\hline $\mathrm{O} 2 \mathrm{C}$ & $\mathrm{C} 1 \mathrm{C}$ & $\mathrm{C} 2 \mathrm{C}$ & F5C & $163.0(10)$ \\
\hline $\mathrm{O} 2 \mathrm{C}$ & $\mathrm{C} 1 \mathrm{C}$ & $\mathrm{C} 2 \mathrm{C}$ & F6C & $99.7(8)$ \\
\hline O1D & C1D & $\mathrm{C} 2 \mathrm{D}$ & F1D & $-160.5(14)$ \\
\hline O1D & C1D & $\mathrm{C} 2 \mathrm{D}$ & F2D & $175.2(16)$ \\
\hline O1D & C1D & $\mathrm{C} 2 \mathrm{D}$ & F3D & $93.2(6)$ \\
\hline O1D & C1D & $\mathrm{C} 2 \mathrm{D}$ & F4D & $0.3(10)$ \\
\hline O1D & C1D & $\mathrm{C} 2 \mathrm{D}$ & F5D & $-23.1(13)$ \\
\hline O1D & C1D & $\mathrm{C} 2 \mathrm{D}$ & F6D & $-95.0(6)$ \\
\hline $\mathrm{O} 2 \mathrm{D}$ & C1D & $\mathrm{C} 2 \mathrm{D}$ & F1D & $23.8(15)$ \\
\hline $\mathrm{O} 2 \mathrm{D}$ & C1D & $\mathrm{C} 2 \mathrm{D}$ & $\mathrm{F} 2 \mathrm{D}$ & $-0.5(18)$ \\
\hline $\mathrm{O} 2 \mathrm{D}$ & C1D & $\mathrm{C} 2 \mathrm{D}$ & F3D & $-82.6(6)$ \\
\hline $\mathrm{O} 2 \mathrm{D}$ & C1D & $\mathrm{C} 2 \mathrm{D}$ & F4D & $-175.4(7)$ \\
\hline $\mathrm{O} 2 \mathrm{D}$ & C1D & $\mathrm{C} 2 \mathrm{D}$ & F5D & $161.2(10)$ \\
\hline $\mathrm{O} 2 \mathrm{D}$ & C1D & $\mathrm{C} 2 \mathrm{D}$ & F6D & $89.3(6)$ \\
\hline O1B & C1B & $\mathrm{C} 2 \mathrm{~B}$ & C3B & $-178.7(2)$ \\
\hline $\mathrm{N} 1 \mathrm{~B}$ & C1B & $\mathrm{C} 2 \mathrm{~B}$ & $\mathrm{C} 3 \mathrm{~B}$ & $0.0(4)$ \\
\hline $\mathrm{C} 1 \mathrm{~B}$ & N1B & C5B & C4B & $-1.0(4)$ \\
\hline $\mathrm{C} 1 \mathrm{~B}$ & $\mathrm{C} 2 \mathrm{~B}$ & C3B & C4B & $0.5(4)$ \\
\hline $\mathrm{C} 2 \mathrm{~B}$ & $\mathrm{C} 3 \mathrm{~B}$ & C4B & C5B & $-1.1(4)$ \\
\hline $\mathrm{C} 2 \mathrm{~B}$ & C3B & $\mathrm{C} 4 \mathrm{~B}$ & C6B & $178.3(2)$ \\
\hline $\mathrm{C} 3 \mathrm{~B}$ & $\mathrm{C} 4 \mathrm{~B}$ & C5B & N1B & $1.4(4)$ \\
\hline $\mathrm{C} 3 \mathrm{~B}$ & $\mathrm{C} 4 \mathrm{~B}$ & C6B & C7B & $29.5(4)$ \\
\hline C3B & $\mathrm{C} 4 \mathrm{~B}$ & C6B & $\mathrm{C} 8 \mathrm{~B}^{\mathrm{i}}$ & $-150.3(2)$ \\
\hline C4B & C6B & C7B & $\mathrm{C} 8 \mathrm{~B}$ & $179.9(2)$ \\
\hline C5B & N1B & C1B & O1B & $179.1(2)$ \\
\hline C5B & N1B & $\mathrm{C} 1 \mathrm{~B}$ & $\mathrm{C} 2 \mathrm{~B}$ & $0.3(4)$ \\
\hline C5B & $\mathrm{C} 4 \mathrm{~B}$ & C6B & C7B & $-151.1(3)$ \\
\hline C5B & C4B & C6B & $\mathrm{C} 8 \mathrm{~B}^{\mathrm{i}}$ & 29.1(4) \\
\hline C6B & $\mathrm{C} 4 \mathrm{~B}$ & C5B & N1B & $-178.0(2)$ \\
\hline C6B & C7B & $\mathrm{C} 8 \mathrm{~B}$ & $\mathrm{C} 6 \mathrm{~B}^{\mathrm{i}}$ & $0.3(4)$ \\
\hline $\mathrm{C} 8 \mathrm{~B}^{\mathrm{i}}$ & C6B & C7B & $\mathrm{C} 8 \mathrm{~B}$ & $-0.3(4)$ \\
\hline O1A & $\mathrm{C} 1 \mathrm{~A}$ & $\mathrm{C} 2 \mathrm{~A}$ & $\mathrm{C} 3 \mathrm{~A}$ & $-178.1(2)$ \\
\hline N1A & $\mathrm{C} 1 \mathrm{~A}$ & $\mathrm{C} 2 \mathrm{~A}$ & $\mathrm{C} 3 \mathrm{~A}$ & $1.5(3)$ \\
\hline $\mathrm{C} 1 \mathrm{~A}$ & N1A & C5A & $\mathrm{C} 4 \mathrm{~A}$ & $-0.7(4)$ \\
\hline $\mathrm{C} 1 \mathrm{~A}$ & $\mathrm{C} 2 \mathrm{~A}$ & $\mathrm{C} 3 \mathrm{~A}$ & $\mathrm{C} 4 \mathrm{~A}$ & $-1.0(4)$ \\
\hline $\mathrm{C} 2 \mathrm{~A}$ & $\mathrm{C} 3 \mathrm{~A}$ & $\mathrm{C} 4 \mathrm{~A}$ & C5A & $-0.4(4)$ \\
\hline $\mathrm{C} 2 \mathrm{~A}$ & $\mathrm{C} 3 \mathrm{~A}$ & $\mathrm{C} 4 \mathrm{~A}$ & C6A & $-179.0(2)$ \\
\hline C3A & $\mathrm{C} 4 \mathrm{~A}$ & C5A & N1A & $1.3(4)$ \\
\hline $\mathrm{C} 3 \mathrm{~A}$ & $\mathrm{C} 4 \mathrm{~A}$ & C6A & C7A & $32.0(3)$ \\
\hline C3A & $\mathrm{C} 4 \mathrm{~A}$ & C6A & $\mathrm{C} 8 \mathrm{~A}^{\mathrm{ii}}$ & $-149.9(2)$ \\
\hline $\mathrm{C} 4 \mathrm{~A}$ & C6A & C7A & $\mathrm{C} 8 \mathrm{~A}$ & $178.1(2)$ \\
\hline C5A & N1A & $\mathrm{C} 1 \mathrm{~A}$ & O1A & $179.0(2)$ \\
\hline C5A & N1A & $\mathrm{C} 1 \mathrm{~A}$ & $\mathrm{C} 2 \mathrm{~A}$ & $-0.7(3)$ \\
\hline C5A & $\mathrm{C} 4 \mathrm{~A}$ & C6A & $\mathrm{C} 7 \mathrm{~A}$ & $-146.6(3)$ \\
\hline C5A & $\mathrm{C} 4 \mathrm{~A}$ & C6A & $\mathrm{C} 8 \mathrm{~A}^{\mathrm{ii}}$ & $31.5(4)$ \\
\hline C6A & $\mathrm{C} 4 \mathrm{~A}$ & C5A & N1A & $179.9(2)$ \\
\hline
\end{tabular}




\begin{tabular}{|c|c|c|}
\hline C6A & C7A & C8A \\
\hline $\mathrm{C} 8 \mathrm{~A}^{\mathrm{ii}}$ & C6A & C7A \\
\hline
\end{tabular}

Symmetry codes: (i) $-\mathrm{x}+3,-\mathrm{y}+2,-\mathrm{z}+1$; (ii) $-\mathrm{x},-\mathrm{y}+2,-\mathrm{z}$.

Table S5. Bond angle $\left({ }^{0}\right)$ observed in $\mathbf{3}$.

\begin{tabular}{|c|c|c|c|}
\hline Atom & Atom & Atom & Angle $/^{\circ}$ \\
\hline C9 & $\mathrm{C} 10$ & C81 & $122.0(2)$ \\
\hline N9 & $\mathrm{C} 10$ & $C 8^{i}$ & $122.0(2)$ \\
\hline $\mathrm{O} 1$ & $\mathrm{C} 2$ & N3 & $119.8(2)$ \\
\hline $\mathrm{O} 1$ & $\mathrm{C} 2$ & $\mathrm{C} 7$ & $125.4(2)$ \\
\hline N3 & $\mathrm{C} 2$ & $\mathrm{C} 7$ & $114.9(2)$ \\
\hline $\mathrm{C} 4$ & N3 & $\mathrm{C} 2$ & $124.49(18)$ \\
\hline N3 & $\mathrm{C} 4$ & C5 & $121.3(2)$ \\
\hline $\mathrm{C} 4$ & $\mathrm{C} 5$ & C6 & $116.5(2)$ \\
\hline $\mathrm{C} 4$ & $\mathrm{C} 5$ & $\mathrm{C} 8$ & $121.7(2)$ \\
\hline C6 & $\mathrm{C} 5$ & $\mathrm{C} 8$ & $121.79(19)$ \\
\hline $\mathrm{C} 7$ & C6 & $\mathrm{C} 5$ & $121.9(2)$ \\
\hline C6 & $\mathrm{C} 7$ & $\mathrm{C} 2$ & $121.0(2)$ \\
\hline N9 & $\mathrm{C} 8$ & $\mathrm{C} 10^{\mathrm{i}}$ & $118.7(2)$ \\
\hline C9 & $\mathrm{C} 8$ & $\mathrm{C} 5$ & $118.8(2)$ \\
\hline N9 & $\mathrm{C} 8$ & C5 & $118.8(2)$ \\
\hline $\mathrm{C} 10^{\mathrm{i}}$ & $\mathrm{C} 8$ & C5 & $122.44(19)$ \\
\hline C8 & N9 & $\mathrm{C} 10$ & $119.3(2)$ \\
\hline $\mathrm{C} 8$ & $\mathrm{C} 9$ & $\mathrm{C} 10$ & $119.3(2)$ \\
\hline
\end{tabular}

Symmetry codes: (i) $-\mathrm{x},-\mathrm{y}+1,-\mathrm{z}+1$.

Table S6. Bond length $(\AA)$ observed in $\mathbf{3}$.

\begin{tabular}{lll} 
Atom & Atom & Length/A \\
\hline O1 & C2 & $1.253(3)$ \\
C10 & C9 & $1.361(3)$ \\
C10 & N9 & $1.361(3)$ \\
C10 & C8 & $1.383(3)$ \\
C2 & N3 & $1.368(3)$ \\
C2 & C7 & $1.424(3)$ \\
C4 & C5 & $1.364(3)$ \\
C5 & C6 & $1.413(3)$ \\
C5 & C8 & $1.485(3)$ \\
C6 & C7 & $1.357(3)$ \\
C8 & C9 & $1.359(3)$ \\
C8 & N9 & $1.359(3)$ \\
\hline Sym
\end{tabular}

Symmetry codes: (i) $-\mathrm{x},-\mathrm{y}+1,-\mathrm{z}+1$. 
Table S7. Hydrogen bond geometry $\left(\AA^{0},{ }^{0}\right)$ observed in 4.

\begin{tabular}{lllll}
$\boldsymbol{D}-\boldsymbol{H} \cdots \boldsymbol{A}$ & $\boldsymbol{D}-\boldsymbol{H}$ & $\boldsymbol{H} \cdots \boldsymbol{A}$ & $\boldsymbol{D} \cdots \boldsymbol{A}$ & $\boldsymbol{D}-\boldsymbol{H} \cdots \boldsymbol{A}$ \\
\hline $\mathrm{O} 1-\mathrm{H} 1 \cdots \mathrm{O} 4$ & $0.830(2)$ & $1.669(11)$ & $2.481(3)$ & $165(4)$ \\
$\mathrm{O} 2-\mathrm{H} 2 \cdots \mathrm{O} 6$ & $0.830(2)$ & $1.645(13)$ & $2.454(3)$ & $164(5)$ \\
N1-H1A $\cdots \mathrm{O}^{\mathrm{i}}$ & 0.88 & 1.83 & $2.698(3)$ & 170.5 \\
N4-H4 $\cdots$ O5 $^{\mathrm{ii}}$ & 0.88 & 1.94 & $2.798(3)$ & 163.9 \\
\hline
\end{tabular}

Symmetry codes: (i) $-\mathrm{x}-1 / 2, \mathrm{y}+1 / 2,-\mathrm{z}+1 / 2$; (ii) $-\mathrm{x}+3 / 2, \mathrm{y}-1 / 2,-\mathrm{z}+3 / 2$.

Table S8. Bond angle $\left({ }^{0}\right)$ observed in 4 .

\begin{tabular}{|c|c|c|c|}
\hline Atom & Atom & Atom & Angle $/^{\circ}$ \\
\hline $\mathrm{C} 1$ & N1 & $\mathrm{C} 5$ & $123.2(2)$ \\
\hline $\mathrm{C} 8$ & $\mathrm{~N} 2$ & C6 & $117.0(2)$ \\
\hline $\mathrm{C} 7$ & N3 & C9 & $117.1(2)$ \\
\hline C13 & N4 & $\mathrm{C} 14$ & $123.2(2)$ \\
\hline $\mathrm{O} 1$ & $\mathrm{C} 1$ & N1 & $114.7(2)$ \\
\hline $\mathrm{O} 1$ & $\mathrm{C} 1$ & $\mathrm{C} 2$ & $127.2(2)$ \\
\hline N1 & $\mathrm{C} 1$ & $\mathrm{C} 2$ & $118.1(2)$ \\
\hline C3 & $\mathrm{C} 2$ & $\mathrm{C} 1$ & $119.6(2)$ \\
\hline $\mathrm{C} 2$ & $\mathrm{C} 3$ & $\mathrm{C} 4$ & $120.8(3)$ \\
\hline C3 & $\mathrm{C} 4$ & C6 & $123.4(2)$ \\
\hline $\mathrm{C} 5$ & $\mathrm{C} 4$ & $\mathrm{C} 3$ & $117.2(2)$ \\
\hline C5 & $\mathrm{C} 4$ & C6 & $119.3(2)$ \\
\hline N1 & $\mathrm{C} 5$ & $\mathrm{C} 4$ & $121.0(2)$ \\
\hline $\mathrm{N} 2$ & C6 & $\mathrm{C} 4$ & $115.9(2)$ \\
\hline $\mathrm{N} 2$ & C6 & $\mathrm{C} 7$ & $120.4(2)$ \\
\hline $\mathrm{C} 7$ & C6 & $\mathrm{C} 4$ & $123.6(2)$ \\
\hline N3 & $\mathrm{C} 7$ & C6 & $122.7(2)$ \\
\hline $\mathrm{N} 2$ & $\mathrm{C} 8$ & C9 & $122.4(2)$ \\
\hline N3 & $\mathrm{C} 9$ & $\mathrm{C} 8$ & $120.5(2)$ \\
\hline N3 & $\mathrm{C} 9$ & $\mathrm{C} 10$ & $116.0(2)$ \\
\hline $\mathrm{C} 8$ & C9 & $\mathrm{C} 10$ & $123.5(2)$ \\
\hline C11 & $\mathrm{C} 10$ & C9 & $123.8(2)$ \\
\hline C14 & $\mathrm{C} 10$ & C9 & $119.0(2)$ \\
\hline C14 & $\mathrm{C} 10$ & $\mathrm{C} 11$ & $117.1(2)$ \\
\hline C12 & $\mathrm{C} 11$ & $\mathrm{C} 10$ & $121.0(3)$ \\
\hline C11 & $\mathrm{C} 12$ & C13 & $120.0(3)$ \\
\hline $\mathrm{O} 2$ & $\mathrm{C} 13$ & N4 & $115.0(2)$ \\
\hline $\mathrm{O} 2$ & $\mathrm{C} 13$ & $\mathrm{C} 12$ & $127.4(2)$ \\
\hline $\mathrm{N} 4$ & $\mathrm{C} 13$ & $\mathrm{C} 12$ & $117.6(2)$ \\
\hline $\mathrm{N} 4$ & $\mathrm{C} 14$ & $\mathrm{C} 10$ & $121.1(2)$ \\
\hline $\mathrm{O} 3$ & C15 & $\mathrm{O} 4$ & $128.3(3)$ \\
\hline $\mathrm{O} 3$ & $\mathrm{C} 15$ & C16 & $115.2(3)$ \\
\hline $\mathrm{O} 4$ & C15 & C16 & $116.5(3)$ \\
\hline $\mathrm{F} 1$ & C16 & C15 & $117.0(3)$ \\
\hline $\mathrm{F} 1$ & $\mathrm{C} 16$ & $\mathrm{~F} 2$ & $109.3(4)$ \\
\hline
\end{tabular}




\begin{tabular}{llll} 
F1 & C16 & F3 & $110.6(4)$ \\
F2 & C16 & C15 & $108.7(3)$ \\
F3 & C16 & C15 & $110.1(3)$ \\
F3 & C16 & F2 & $99.7(4)$ \\
F1A & C16 & C15 & $106.9(5)$ \\
F2A & C16 & C15 & $107.8(5)$ \\
F2A & C16 & F1A & $97.9(8)$ \\
F3A & C16 & C15 & $120.9(5)$ \\
F3A & C16 & F1A & $108.5(9)$ \\
F3A & C16 & F2A & $112.3(9)$ \\
O5 & C17 & O6 & $129.1(3)$ \\
O5 & C17 & C18 & $118.0(3)$ \\
O6 & C17 & C17 & $113.0(2)$ \\
F4 & C18 & C17 & $112.2(13)$ \\
F5 & C18 & F4 & $114.7(9)$ \\
F5 & C18 & F6 & $103.8(15)$ \\
F5 & C18 & C17 & $109.3(16)$ \\
F6 & C18 & F4 & $112.0(16)$ \\
F6 & C18 & C17 & $104.1(17)$ \\
F4A & C18 & C17 & $109.8(5)$ \\
F5A & C18 & F4A & $111.8(4)$ \\
F5A & C18 & F6A & $109.7(6)$ \\
F5A & C18 & C17 & $109.1(5)$ \\
F6A & C18 & F4A & $111.4(3)$ \\
F6A & C18 & & $104.7(4)$ \\
\hline
\end{tabular}

Table S9. Bond length $(\AA)$ observed in 4 .

\begin{tabular}{lll} 
Atom & Atom & Length/A \\
\hline O1 & C1 & $1.308(3)$ \\
O2 & C13 & $1.298(3)$ \\
O3 & C15 & $1.215(4)$ \\
O4 & C15 & $1.239(3)$ \\
O5 & C17 & $1.228(3)$ \\
O6 & C17 & $1.254(4)$ \\
$\mathrm{N} 1$ & $\mathrm{C} 1$ & $1.342(3)$ \\
$\mathrm{N} 1$ & $\mathrm{C} 5$ & $1.354(3)$ \\
$\mathrm{N} 2$ & $\mathrm{C} 6$ & $1.341(3)$ \\
$\mathrm{N} 2$ & $\mathrm{C} 8$ & $1.337(3)$ \\
$\mathrm{N} 3$ & $\mathrm{C} 7$ & $1.331(3)$ \\
$\mathrm{N} 3$ & $\mathrm{C} 9$ & $1.340(3)$ \\
$\mathrm{N} 4$ & $\mathrm{C} 13$ & $1.351(3)$ \\
$\mathrm{N} 4$ & $\mathrm{C} 14$ & $1.353(3)$ \\
$\mathrm{C} 1$ & $\mathrm{C} 2$ & $1.388(4)$ \\
$\mathrm{C} 2$ & $\mathrm{C} 3$ & $1.380(4)$ \\
$\mathrm{C} 3$ & $\mathrm{C} 4$ & $1.409(3)$ \\
$\mathrm{C} 4$ & $\mathrm{C} 5$ & $1.359(4)$
\end{tabular}




\begin{tabular}{lll} 
C4 & C6 & $1.484(3)$ \\
C6 & C7 & $1.377(4)$ \\
C8 & C9 & $1.380(4)$ \\
C9 & C10 & $1.483(3)$ \\
C10 & C11 & $1.416(3)$ \\
C10 & C14 & $1.363(4)$ \\
C11 & C12 & $1.370(4)$ \\
C12 & C13 & $1.400(4)$ \\
C15 & C16 & $1.535(4)$ \\
C16 & F1 & $1.247(4)$ \\
C16 & F2 & $1.366(5)$ \\
C16 & F3 & $1.344(5)$ \\
C16 & F1A & $1.427(10)$ \\
C16 & F2A & $1.355(10)$ \\
C16 & F3A & $1.119(8)$ \\
C17 118 & $1.544(4)$ \\
C18 & C18 & $1.34(3)$ \\
C18 & F4 & $1.164(19)$ \\
C18 & F5 & $1.19(3)$ \\
C18 & F6 & $1.396(10)$ \\
C18 & F4A & $1.301(9)$ \\
C18 & F5A & $1.359(8)$ \\
\hline
\end{tabular}

Table S10. Torsion Angles $\left({ }^{0}\right)$ observed in 4.

\begin{tabular}{lllll} 
A & B & C & D & Angle ${ }^{\circ}$ \\
\hline O1 & C1 & C2 & C3 & $-178.9(3)$ \\
O3 & C15 & C16 & F1 & $-176.4(5)$ \\
O3 & C15 & C16 & F2 & $-52.1(5)$ \\
O3 & C15 & C16 & F3 & $56.2(5)$ \\
O3 & C15 & C16 & F1A & $127.9(7)$ \\
O3 & C15 & C16 & F2A & $-127.7(7)$ \\
O3 & C15 & C16 & F3A & $3.3(12)$ \\
O4 & C15 & C16 & F1 & $2.5(6)$ \\
O4 & C15 & C16 & F2 & $126.8(4)$ \\
O4 & C15 & C16 & F3 & $-124.9(4)$ \\
O4 & C15 & C16 & F1A & $-53.2(7)$ \\
O4 & C15 & C16 & F2A & $51.2(8)$ \\
O4 & C15 & C16 & F3A & $-177.8(11)$ \\
O5 & C17 & C18 & F4 & $-10.2(11)$ \\
O5 & C17 & C18 & F5 & $107.9(13)$ \\
O5 & C17 & C18 & F6 & $-126.8(14)$ \\
O5 & C17 & C18 & F4A & $-112.2(5)$ \\
O5 & C17 & C18 & F5A & $9.8(8)$ \\
O5 & C17 & C18 & F6A & $132.3(5)$ \\
O6 & C17 & C18 & F4 & $168.9(10)$ \\
O6 & C17 & C18 & F5 & $-73.0(13)$ \\
\end{tabular}




\begin{tabular}{|c|c|c|c|c|}
\hline O6 & $\mathrm{C} 17$ & C18 & F6 & $52.3(15)$ \\
\hline O6 & $\mathrm{C} 17$ & C18 & $\mathrm{F} 4 \mathrm{~A}$ & $66.9(5)$ \\
\hline O6 & $\mathrm{C} 17$ & C18 & F5A & $-171.1(8)$ \\
\hline O6 & $\mathrm{C} 17$ & C18 & F6A & $-48.6(6)$ \\
\hline N1 & $\mathrm{C} 1$ & $\mathrm{C} 2$ & $\mathrm{C} 3$ & $0.1(5)$ \\
\hline $\mathrm{N} 2$ & C6 & $\mathrm{C} 7$ & N3 & $0.7(5)$ \\
\hline $\mathrm{N} 2$ & $\mathrm{C} 8$ & C9 & N3 & $-1.2(5)$ \\
\hline $\mathrm{N} 2$ & $\mathrm{C} 8$ & C9 & $\mathrm{C} 10$ & $179.1(3)$ \\
\hline N3 & C9 & $\mathrm{C} 10$ & $\mathrm{C} 11$ & $-176.3(3)$ \\
\hline N3 & C9 & $\mathrm{C} 10$ & C14 & $5.5(4)$ \\
\hline $\mathrm{C} 1$ & N1 & $\mathrm{C} 5$ & $\mathrm{C} 4$ & $0.9(5)$ \\
\hline $\mathrm{C} 1$ & $\mathrm{C} 2$ & $\mathrm{C} 3$ & $\mathrm{C} 4$ & $0.5(5)$ \\
\hline $\mathrm{C} 2$ & $\mathrm{C} 3$ & $\mathrm{C} 4$ & $\mathrm{C} 5$ & $-0.4(4)$ \\
\hline $\mathrm{C} 2$ & $\mathrm{C} 3$ & $\mathrm{C} 4$ & C6 & $177.2(3)$ \\
\hline C3 & $\mathrm{C} 4$ & $\mathrm{C} 5$ & $\mathrm{~N} 1$ & $-0.3(4)$ \\
\hline $\mathrm{C} 3$ & $\mathrm{C} 4$ & C6 & $\mathrm{N} 2$ & $174.1(3)$ \\
\hline $\mathrm{C} 3$ & $\mathrm{C} 4$ & C6 & $\mathrm{C} 7$ & $-8.1(5)$ \\
\hline $\mathrm{C} 4$ & C6 & $\mathrm{C} 7$ & N3 & $-177.0(3)$ \\
\hline $\mathrm{C} 5$ & N1 & $\mathrm{C} 1$ & O1 & $178.3(3)$ \\
\hline $\mathrm{C} 5$ & N1 & $\mathrm{C} 1$ & $\mathrm{C} 2$ & $-0.8(5)$ \\
\hline $\mathrm{C} 5$ & $\mathrm{C} 4$ & C6 & $\mathrm{N} 2$ & $-8.3(4)$ \\
\hline $\mathrm{C} 5$ & $\mathrm{C} 4$ & C6 & $\mathrm{C} 7$ & $169.5(3)$ \\
\hline C6 & $\mathrm{N} 2$ & $\mathrm{C} 8$ & C9 & $0.5(4)$ \\
\hline C6 & $\mathrm{C} 4$ & $\mathrm{C} 5$ & N1 & $-178.0(3)$ \\
\hline $\mathrm{C} 7$ & N3 & C9 & $\mathrm{C} 8$ & $1.6(4)$ \\
\hline $\mathrm{C} 7$ & N3 & C9 & $\mathrm{C} 10$ & $-178.7(3)$ \\
\hline $\mathrm{C} 8$ & $\mathrm{~N} 2$ & C6 & $\mathrm{C} 4$ & $177.7(2)$ \\
\hline $\mathrm{C} 8$ & $\mathrm{~N} 2$ & C6 & $\mathrm{C} 7$ & $-0.2(4)$ \\
\hline $\mathrm{C} 8$ & C9 & $\mathrm{C} 10$ & C11 & $3.4(5)$ \\
\hline $\mathrm{C} 8$ & C9 & $\mathrm{C} 10$ & C14 & $-174.7(3)$ \\
\hline C9 & N3 & $\mathrm{C} 7$ & C6 & $-1.4(5)$ \\
\hline C9 & $\mathrm{C} 10$ & $\mathrm{C} 11$ & $\mathrm{C} 12$ & $-179.2(3)$ \\
\hline C9 & $\mathrm{C} 10$ & $\mathrm{C} 14$ & N4 & $179.4(2)$ \\
\hline $\mathrm{C} 10$ & $\mathrm{C} 11$ & $\mathrm{C} 12$ & $\mathrm{C} 13$ & $1.0(5)$ \\
\hline $\mathrm{C} 11$ & $\mathrm{C} 10$ & $\mathrm{C} 14$ & N4 & $1.1(4)$ \\
\hline $\mathrm{C} 11$ & $\mathrm{C} 12$ & $\mathrm{C} 13$ & $\mathrm{O} 2$ & $178.9(3)$ \\
\hline $\mathrm{C} 11$ & $\mathrm{C} 12$ & $\mathrm{C} 13$ & N4 & $-1.0(5)$ \\
\hline $\mathrm{C} 13$ & N4 & $\mathrm{C} 14$ & $\mathrm{C} 10$ & $-1.3(4)$ \\
\hline $\mathrm{C} 14$ & N4 & $\mathrm{C} 13$ & $\mathrm{O} 2$ & $-178.8(3)$ \\
\hline $\mathrm{C} 14$ & N4 & $\mathrm{C} 13$ & $\mathrm{C} 12$ & $1.2(4)$ \\
\hline C14 & $\mathrm{C} 10$ & $\mathrm{C} 11$ & $\mathrm{C} 12$ & $-1.0(4)$ \\
\hline
\end{tabular}




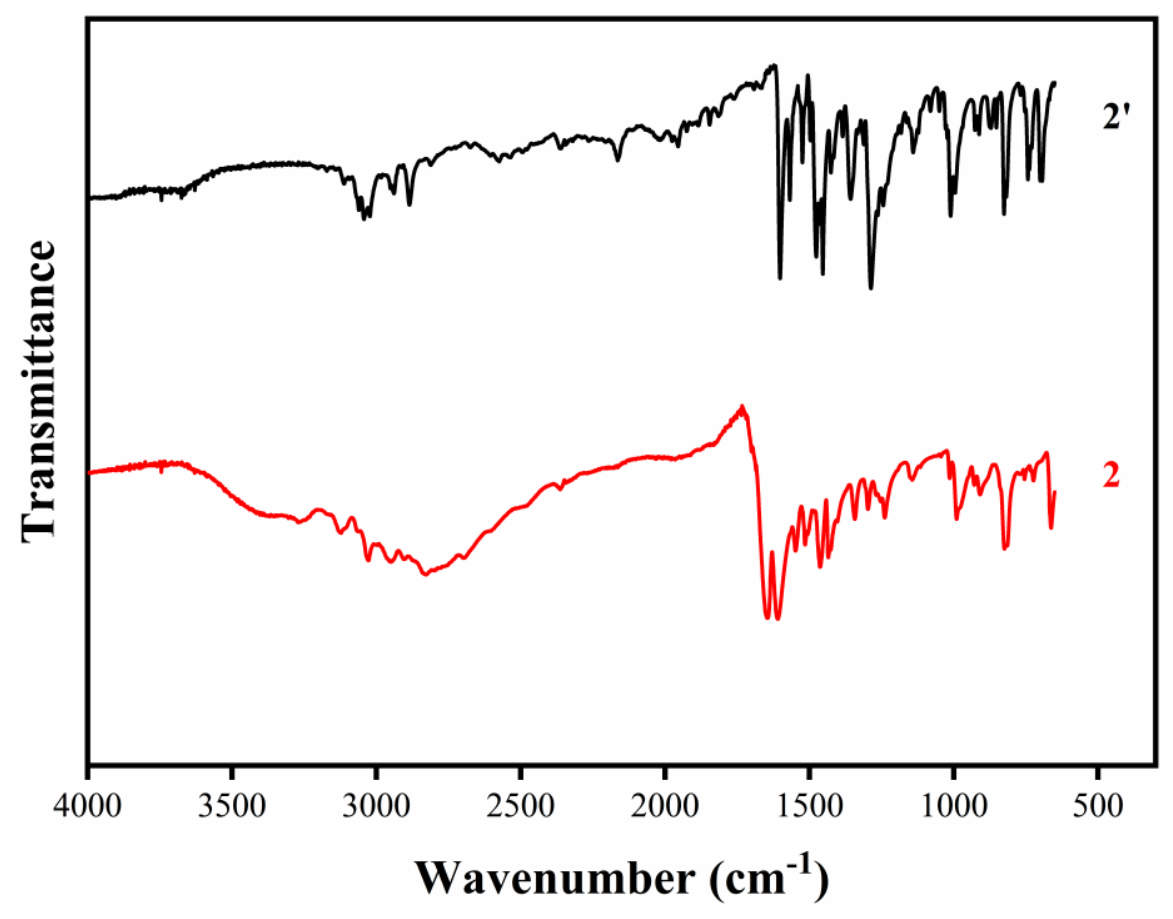

Figure S16. FTIR of compounds 2' (black) and 2 (red).

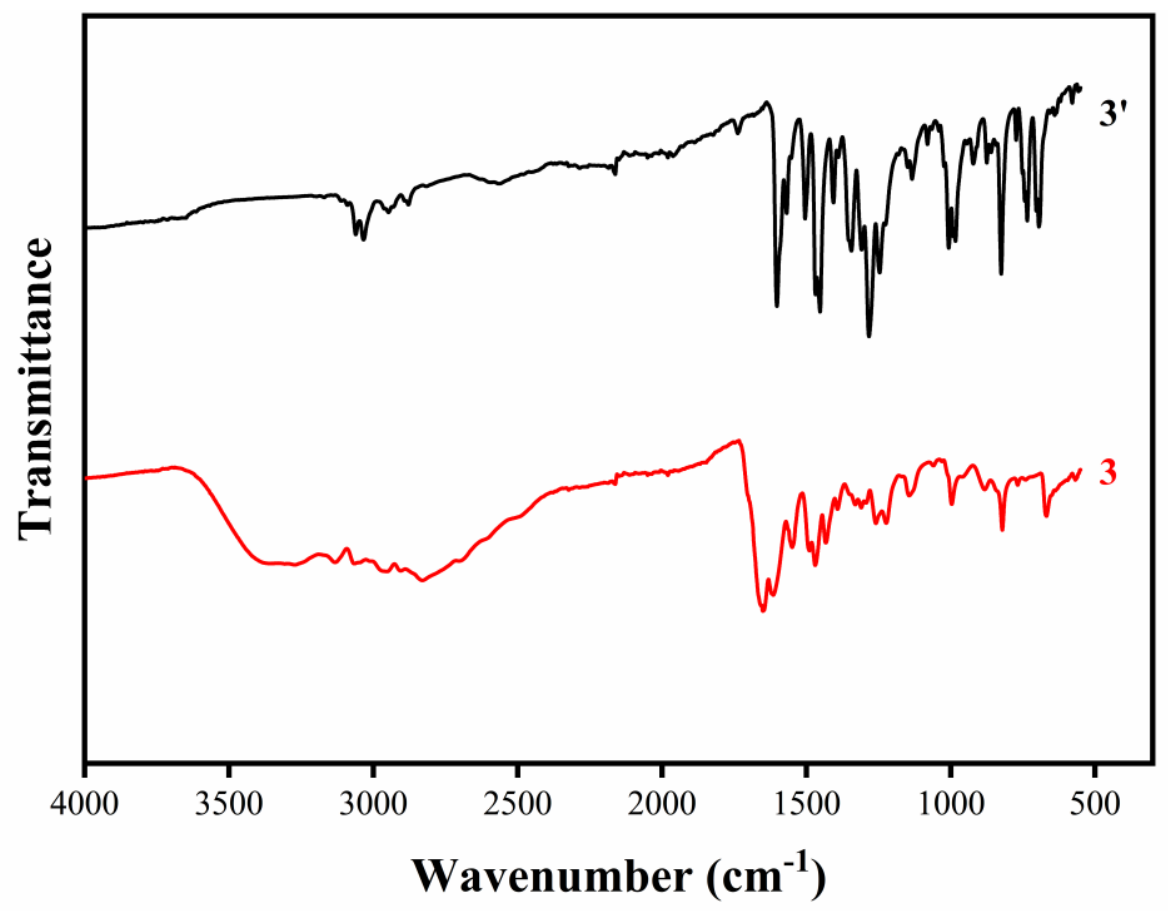

Figure S17. FTIR of compounds 3' (black) and 3 (red). 


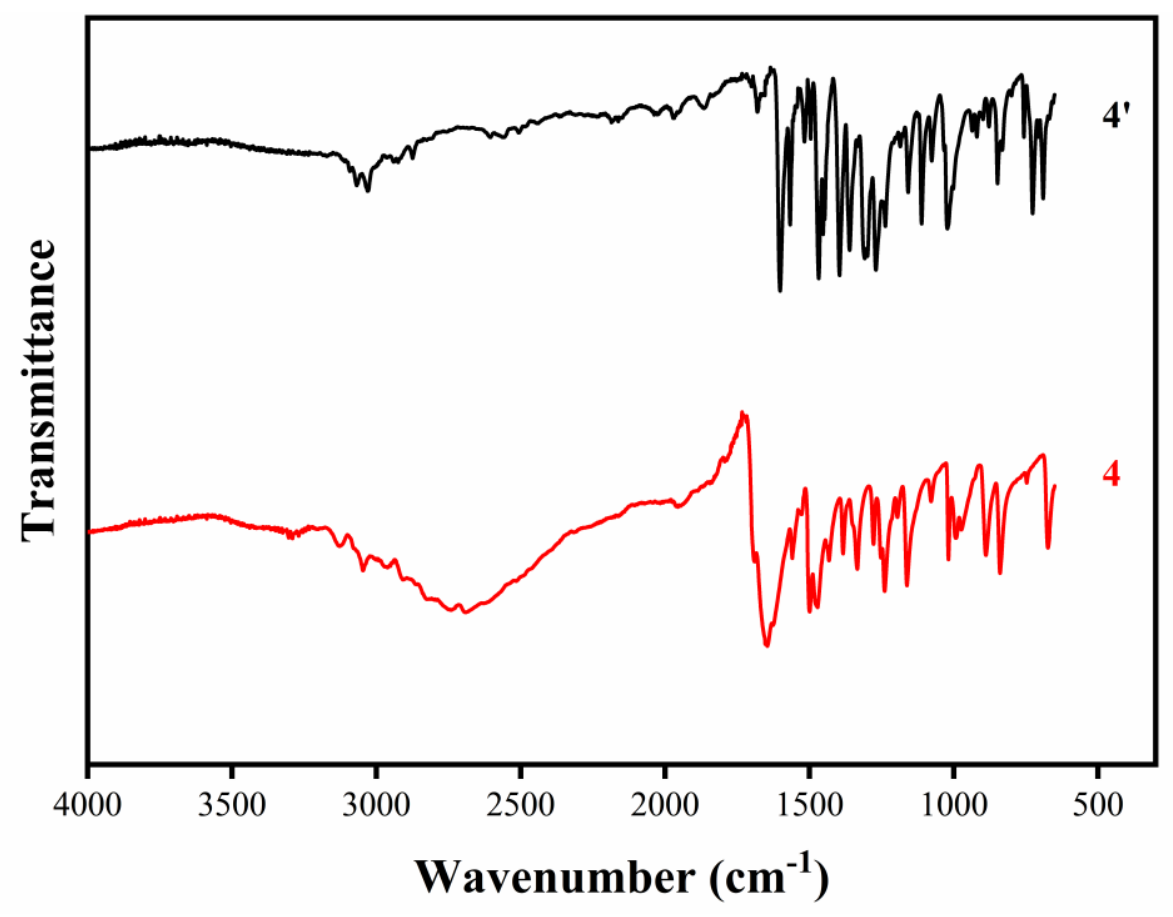

Figure S18. FTIR of compounds 4' (black) and 4 (red).

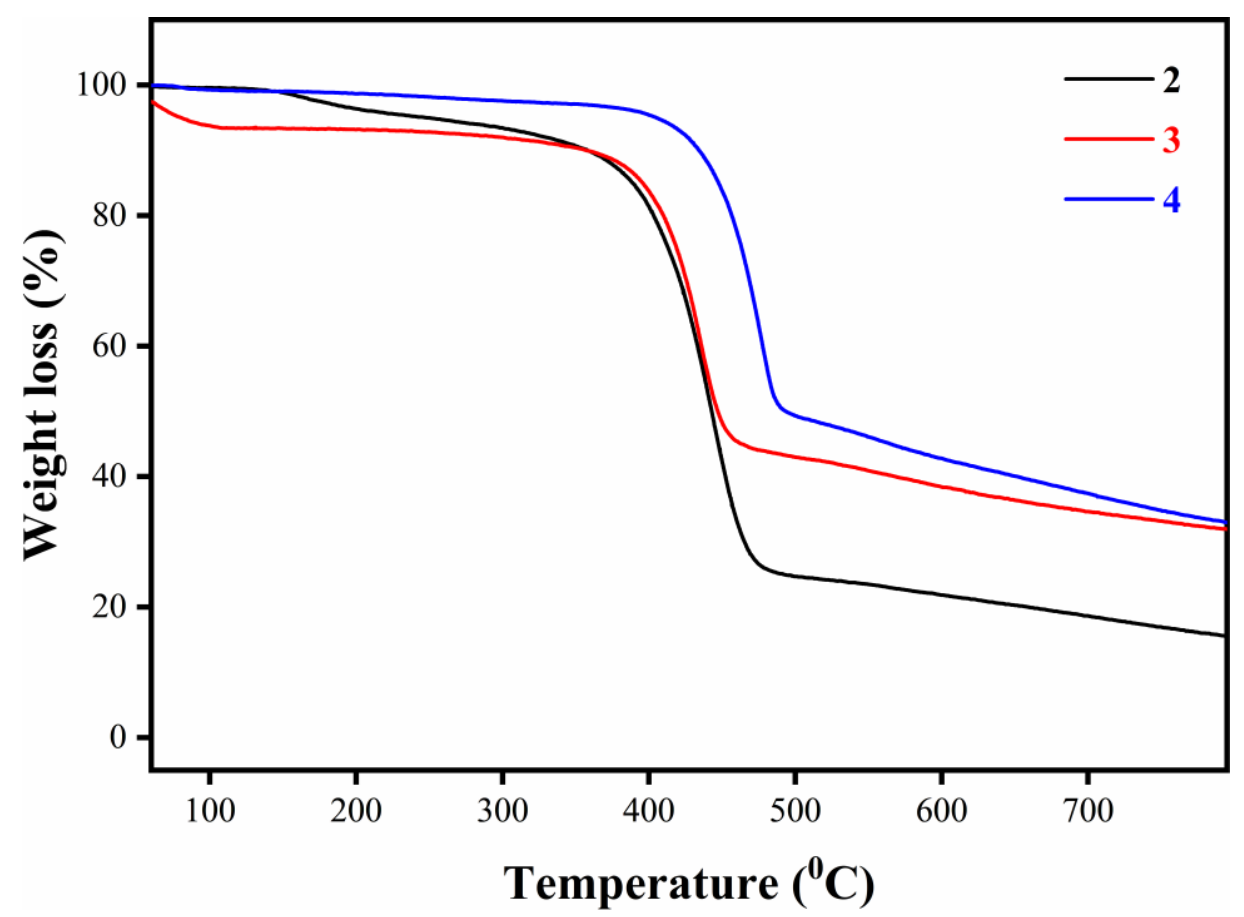

Figure S19. TGA of compounds 2-4. 